\title{
The Impact of Attending a School with High-Achieving Peers: Evidence from the New York City Exam Schools Online Appendix
}

Will Dobbie and Roland G. Fryer, Jr.

October 2013 


\section{Appendix Figure 1 \\ Exam School Eligibility and Baseline Characteristics}

Appx. Figure 1A: Baseline ELA Score

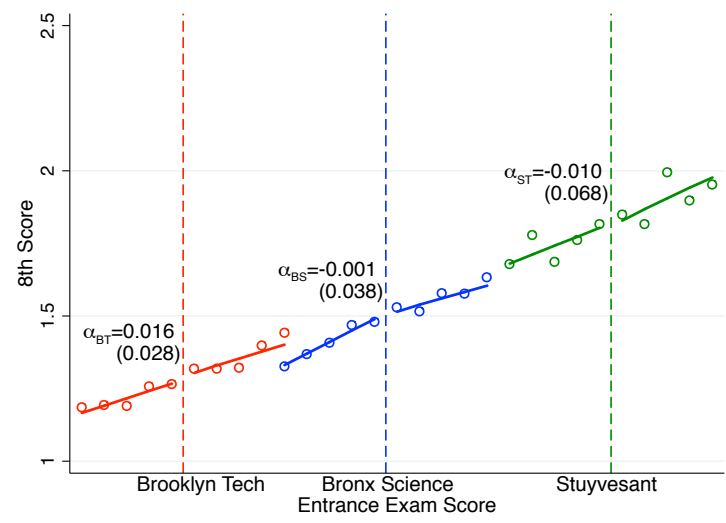

Appx. Figure 1C: Black

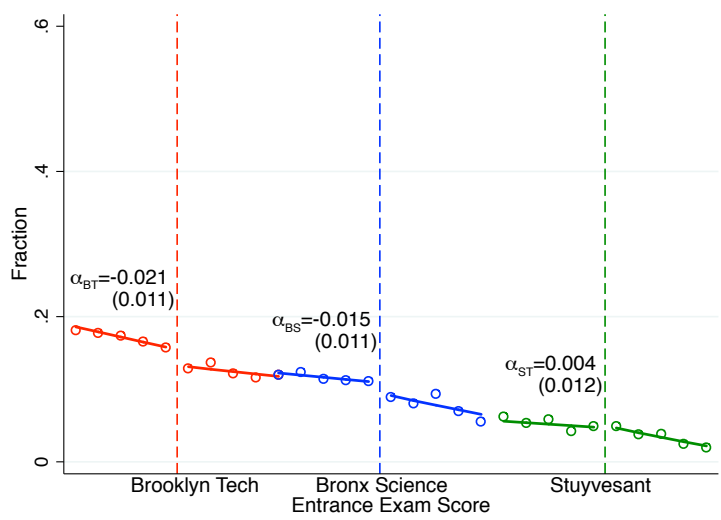

Appx. Figure 1E: Public Middle School

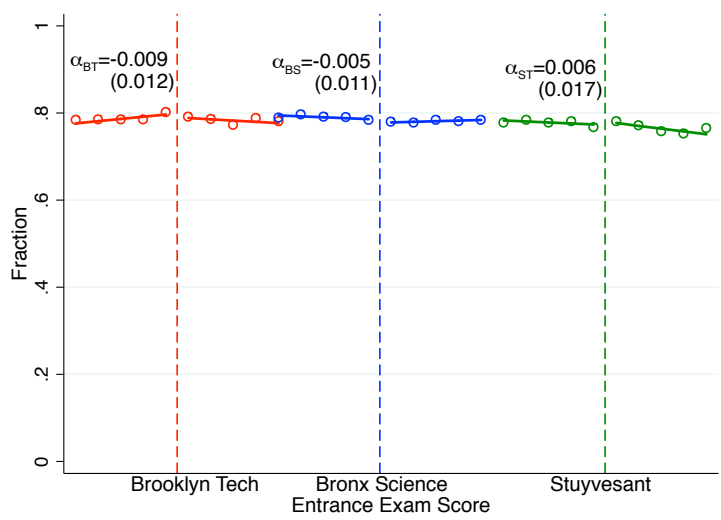

Appx. Figure 1B: Baseline Math Score

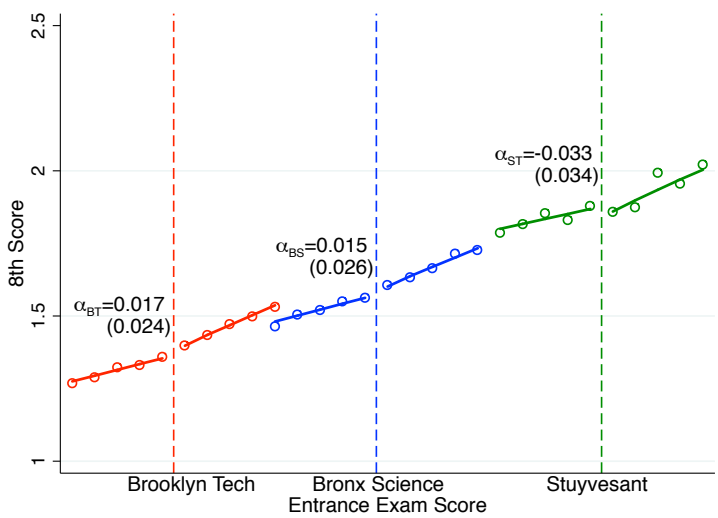

Appx. Figure 1D: Hispanic

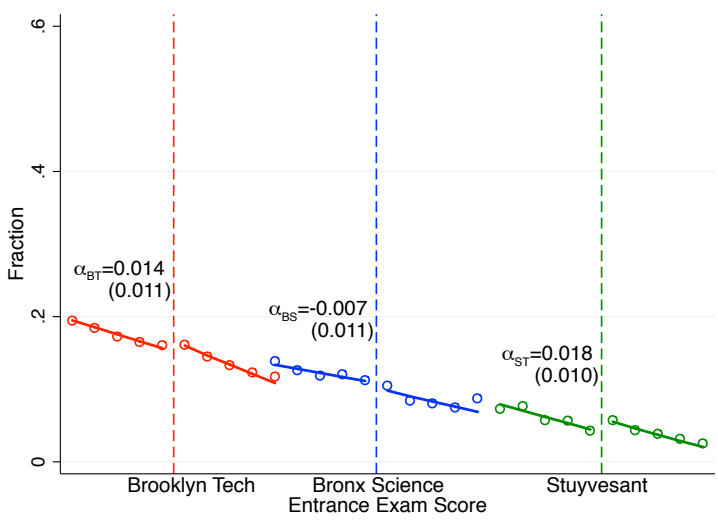

Appx. Figure 1F: Male

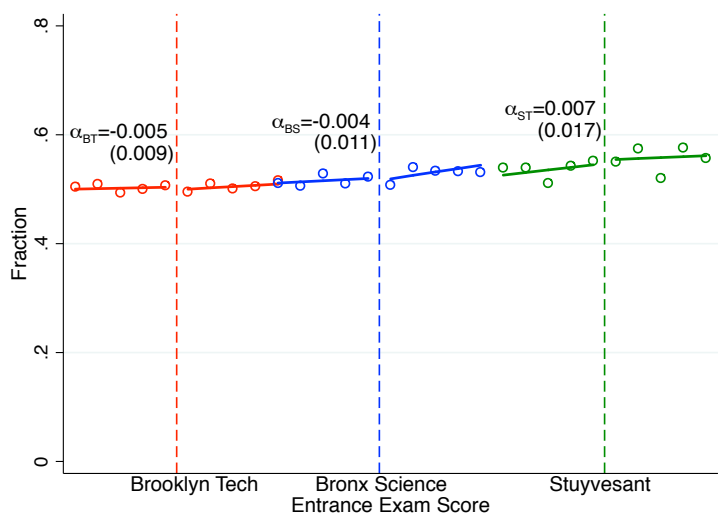

Notes: These figures plot exam school eligibility and baseline characteristics of test takers. The sample for test score outcomes includes exam school applicants in the 2002 - 2013 high school cohorts. The sample for ethnicity includes exam school applicants in the 2008 - 2013 high school cohorts. The sample for gender and middle school type includes exam school applicants in the 1994 - 2013 high school cohorts. The smoothed line in each figure comes from a single local linear regression of each outcome on entrance exam score, school eligibility, and school eligibility interacted with the entrance exam score. Point estimates and standard errors clustered at the exam score level from an analogous regression that also controls for cohort fixed effects are presented next to each eligibility cutoff. See text for additional details. 


\section{Appendix Figure 2 \\ Exam School Eligibility and the Number of Test Takers}

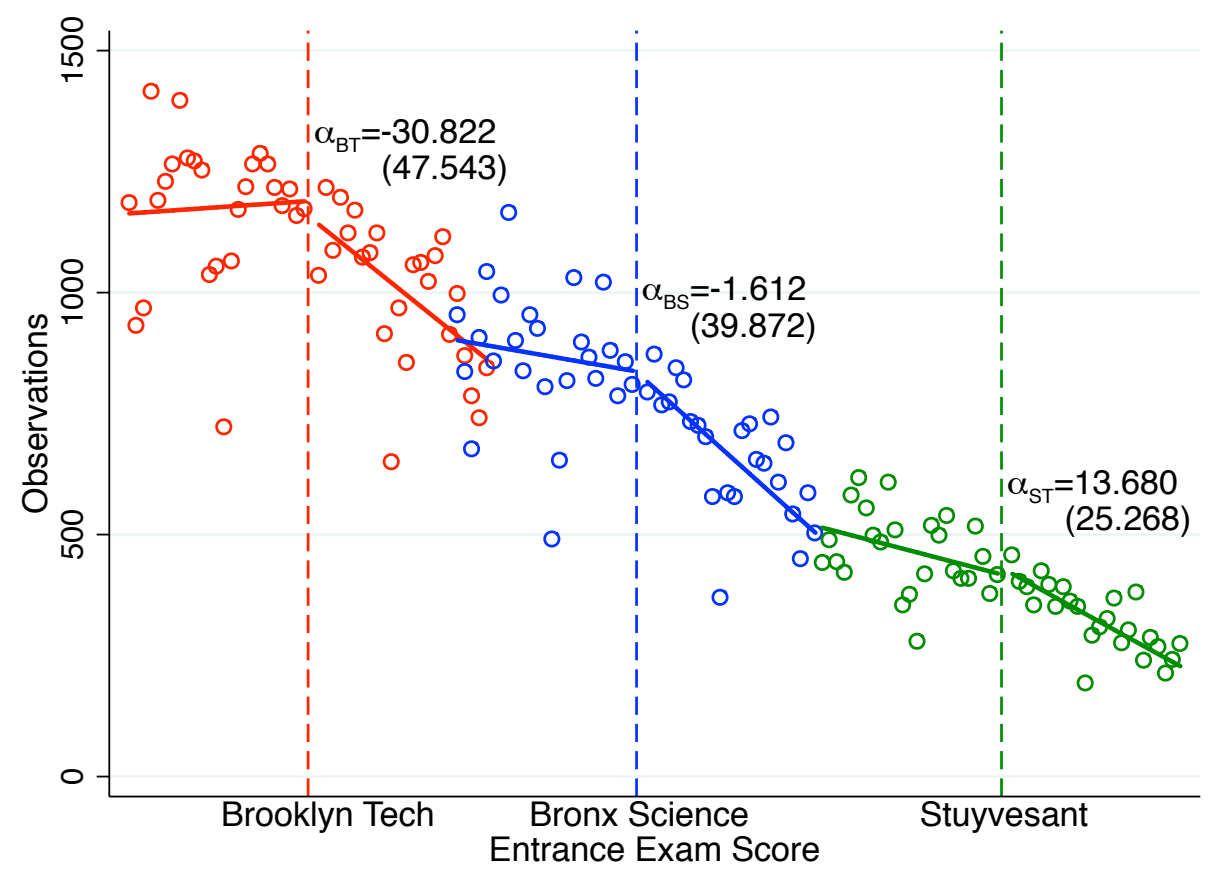

Notes: This figure plots exam school eligibility and the number of test takers. The sample includes exam school applicants in the 1994 - 2013 high school cohorts. The smoothed line in each figure comes from a single local linear regression of each outcome on entrance exam score, school eligibility, and school eligibility interacted with the entrance exam score. Point estimates and standard errors clustered at the exam score level from an analogous regression that also controls for cohort fixed effects are presented next to each eligibility cutoff. See text for additional details. 


\section{Appendix Figure 3 \\ Exam School Eligibility and Graduation from Each School}

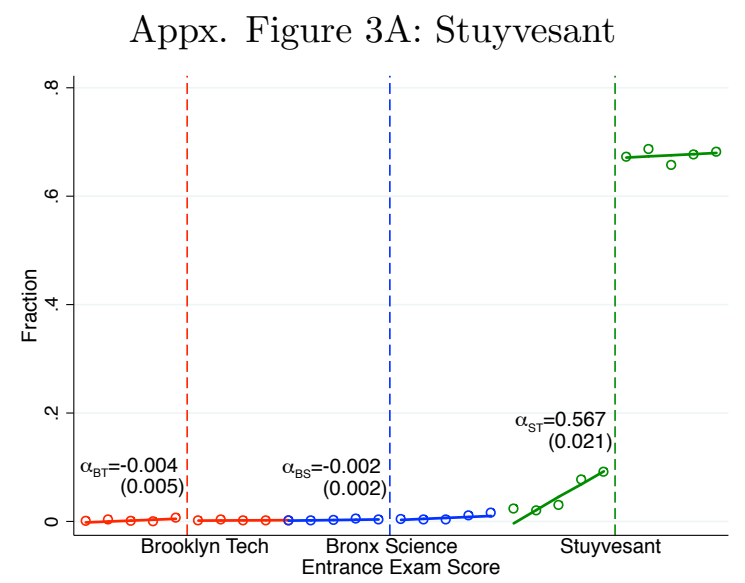

Appx. Figure 3B: Bronx Science

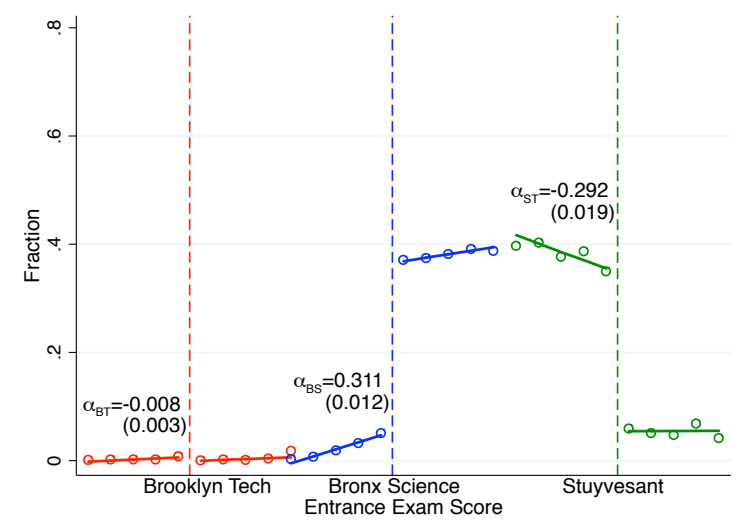

Appx. Figure 3C: Brooklyn Tech

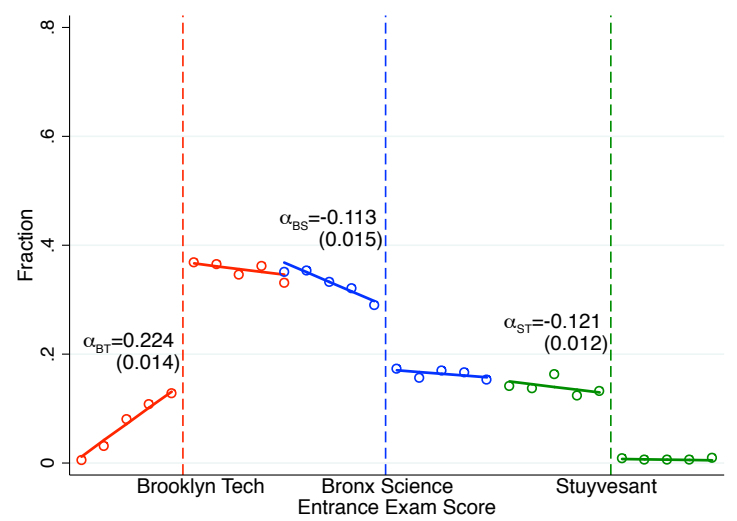

Notes: These figures plot exam school eligibility and the fraction of students graduating from each exam high school. The sample includes exam school applicants in the 2002 - 2009 high school cohorts. The smoothed line in each figure comes from a single local linear regression of each outcome on entrance exam score, school eligibility, and school eligibility interacted with the entrance exam score. Point estimates and standard errors clustered at the exam score level from an analogous regression that also controls for cohort fixed effects are presented next to each eligibility cutoff. The dependent variable in each figure is an indicator for graduating from the indicated high school. See text for additional details. 


\section{Appendix Figure 4 \\ Exam School Eligibility and Enrollment in Any NYC Public High School}

Appx. Figure 4A:

All Applicants

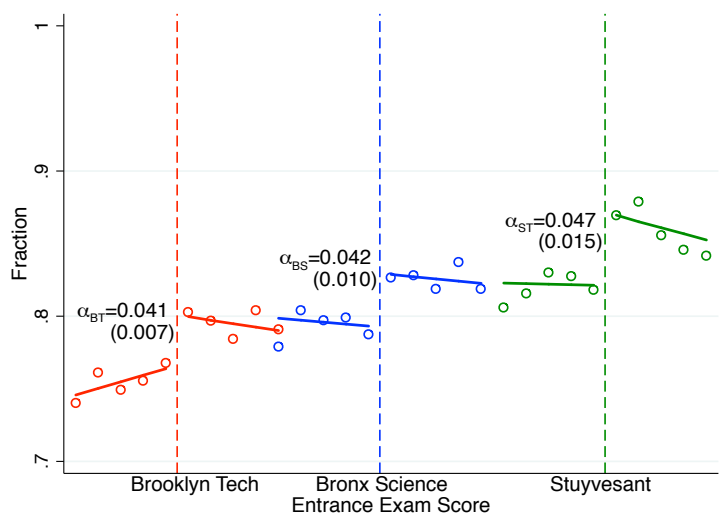

Appx. Figure 4B:

Applicants from Public MS

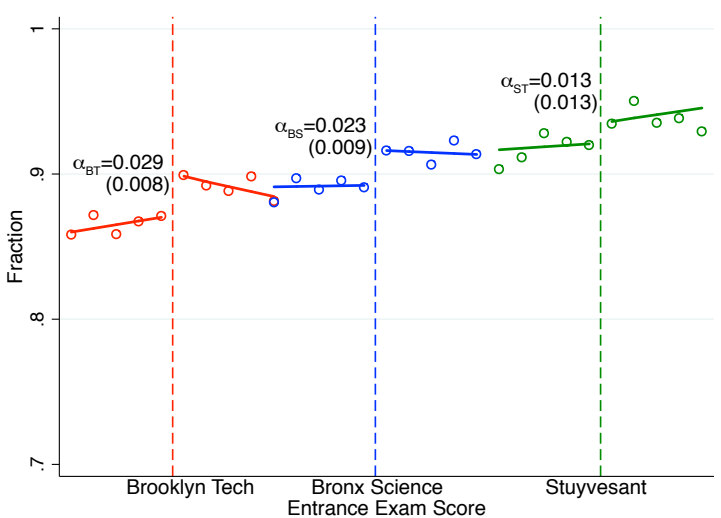

Notes: These figures plot exam school eligibility and the fraction of students enrolling in a NYC public high school. The sample for 1A includes exam school applicants in the $2002-2012$ high school cohorts. The sample for 1B includes exam school applicants in the 2002 - 2012 high school cohorts who attended a NYC public middle school. The smoothed line in each figure comes from a single local linear regression of each outcome on entrance exam score, school eligibility, and school eligibility interacted with the entrance exam score. Point estimates and standard errors clustered at the exam score level from an analogous regression that also controls for cohort fixed effects are presented next to each eligibility cutoff. See text for additional details. 


\section{Appendix Figure 5 \\ Baseline Characteristics of Students Enrolling in Any NYC Public High School}

Appx. Figure 5A: Baseline ELA

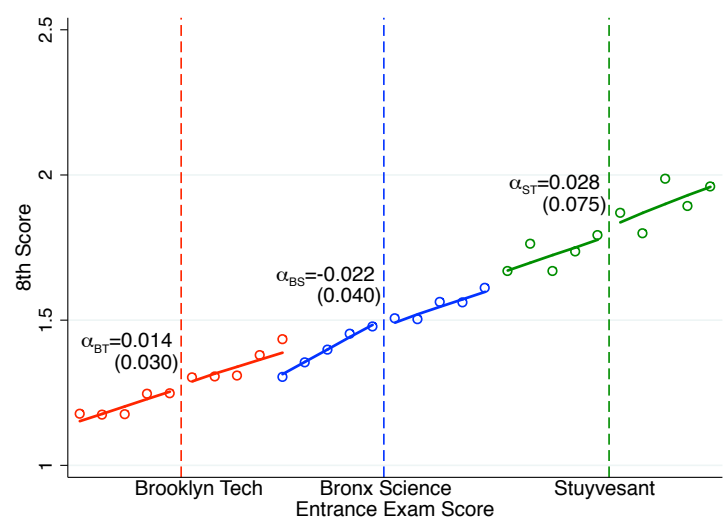

Appx. Figure 5C: Black

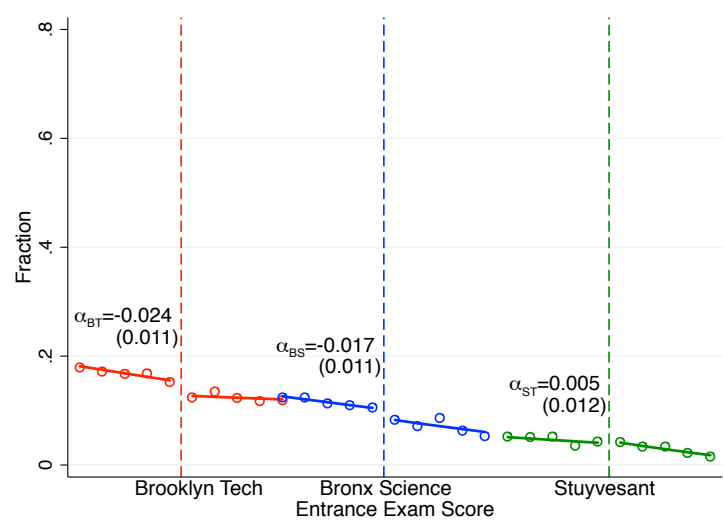

Appx. Figure 5B: Baseline Math

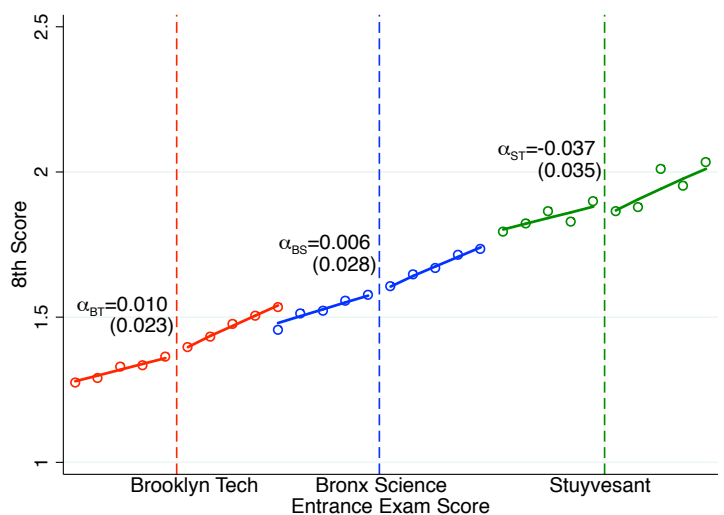

Appx. Figure 5D: Hispanic

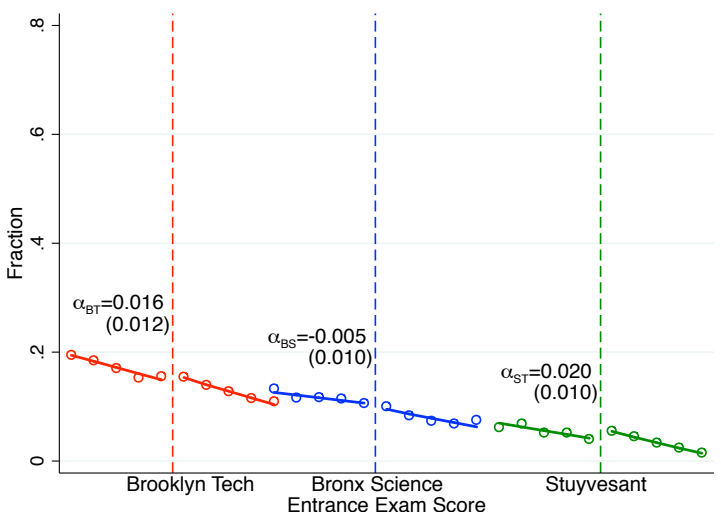

Notes: These figures plot exam school eligibility and the baseline characteristics of students enrolling in a NYC public high school. The sample for test score outcomes includes exam school applicants in the $2002-2012$ high school cohorts. The sample for ethnicity outcomes includes exam school applicants in the 2008 - 2012 high school cohorts. The smoothed line in each figure comes from a single local linear regression of each outcome on entrance exam score, school eligibility, and school eligibility interacted with the entrance exam score. Point estimates and standard errors clustered at the exam score level from an analogous regression that also controls for cohort fixed effects are presented next to each eligibility cutoff. See text for additional details. 


\section{Appendix Figure 6 \\ Exam School Eligibility and Baseline Peer Characteristics in Freshman Courses}

Appx. Figure 6A: Peer ELA Scores

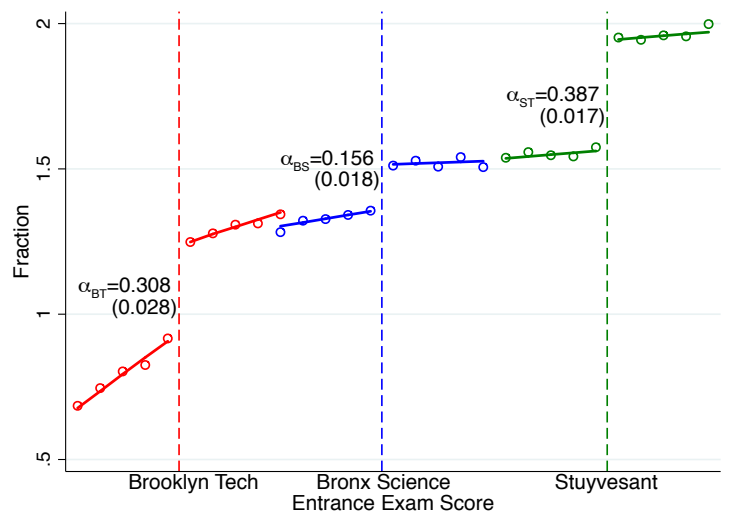

Appx. Figure 6C: Proportion Black

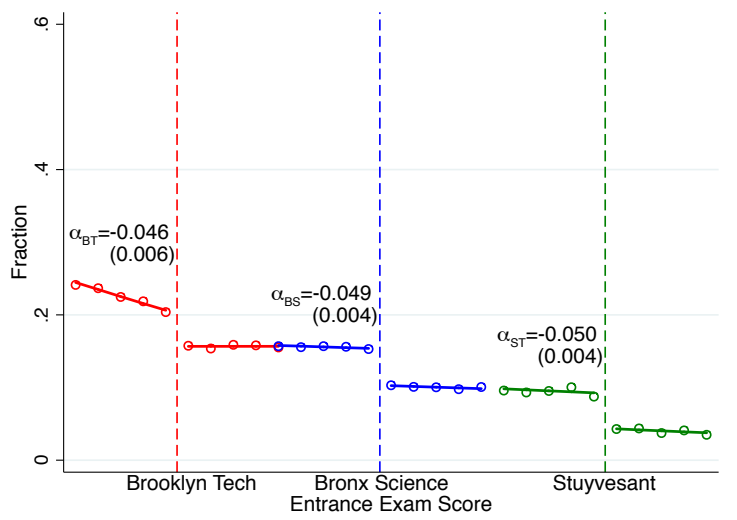

Appx. Figure 6B: Peer Math Scores

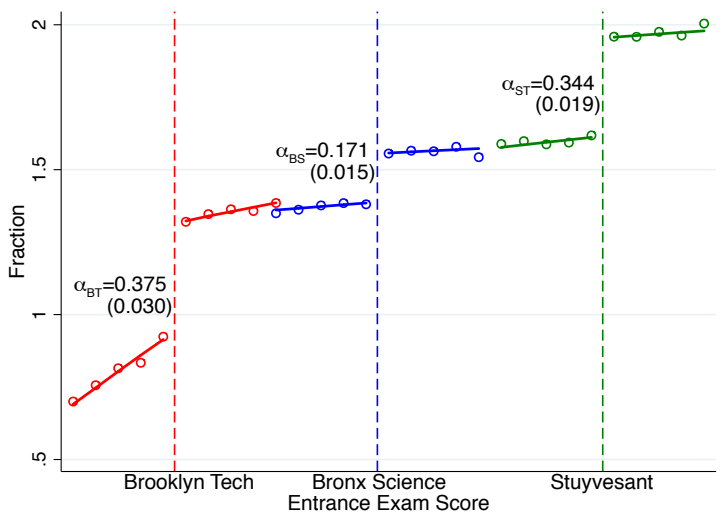

Appx. Figure 6D: Proportion Hispanic

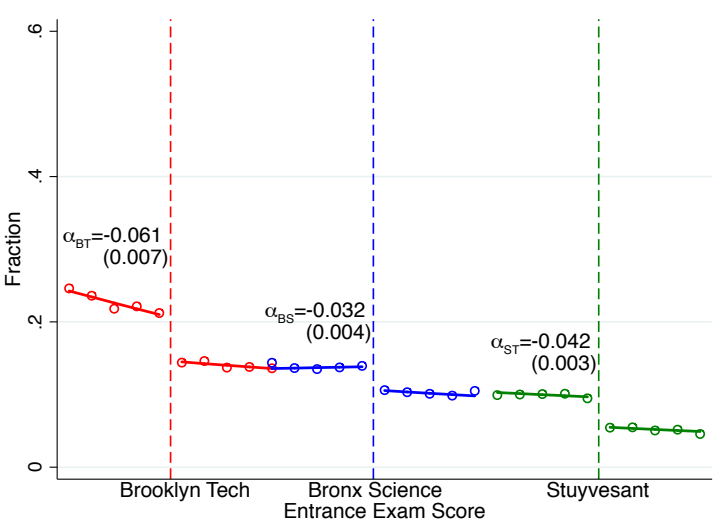

Notes: These figures plot exam school eligibility and average peer characteristics in a student's freshman year courses. The sample includes exam school applicants in the 2002 - 2006 high school cohorts. The smoothed line in each figure comes from a single local linear regression of each outcome on entrance exam score, school eligibility, and school eligibility interacted with the entrance exam score. Point estimates and standard errors clustered at the exam score level from an analogous regression that also controls for cohort fixed effects are presented next to each eligibility cutoff. The dependent variable for each regression is the average peer characteristic in the student's freshman year courses. See text for additional details. 


\section{Appendix Figure 7 \\ Exam School Eligibility and High School Graduation}
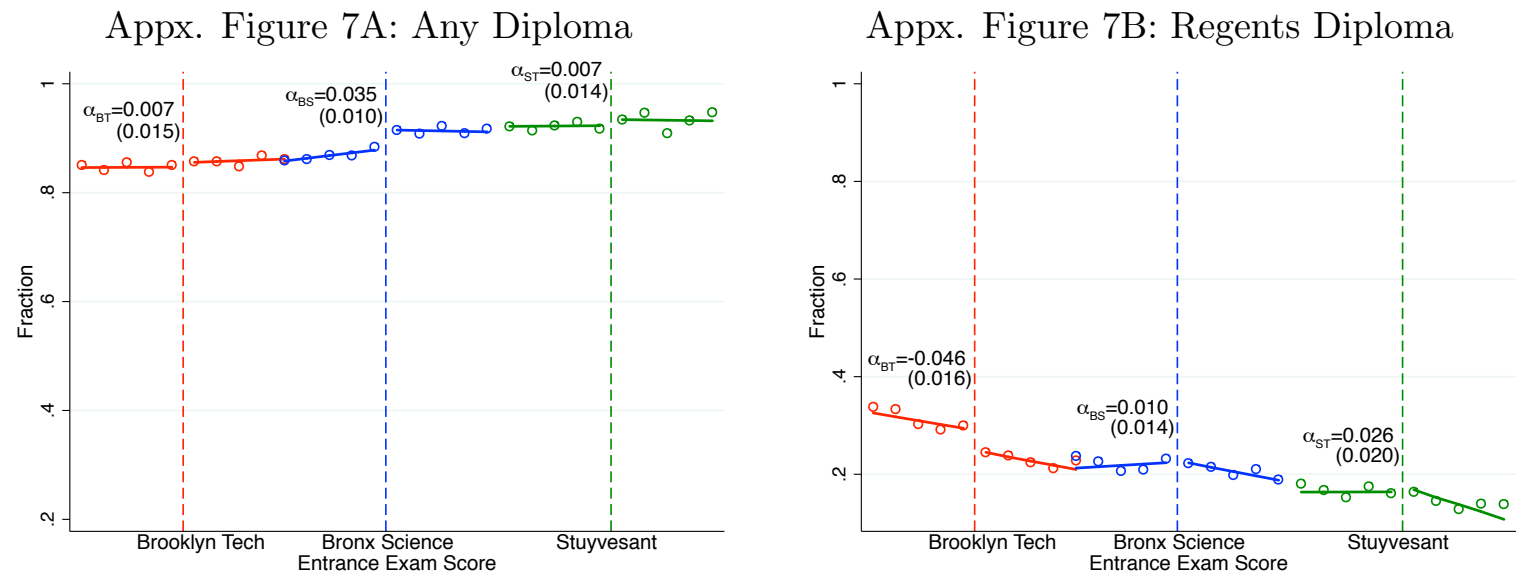

Appx. Figure 7C: Adv. Regents Diploma

Appx. Figure 7D: Regents or Adv. Regents
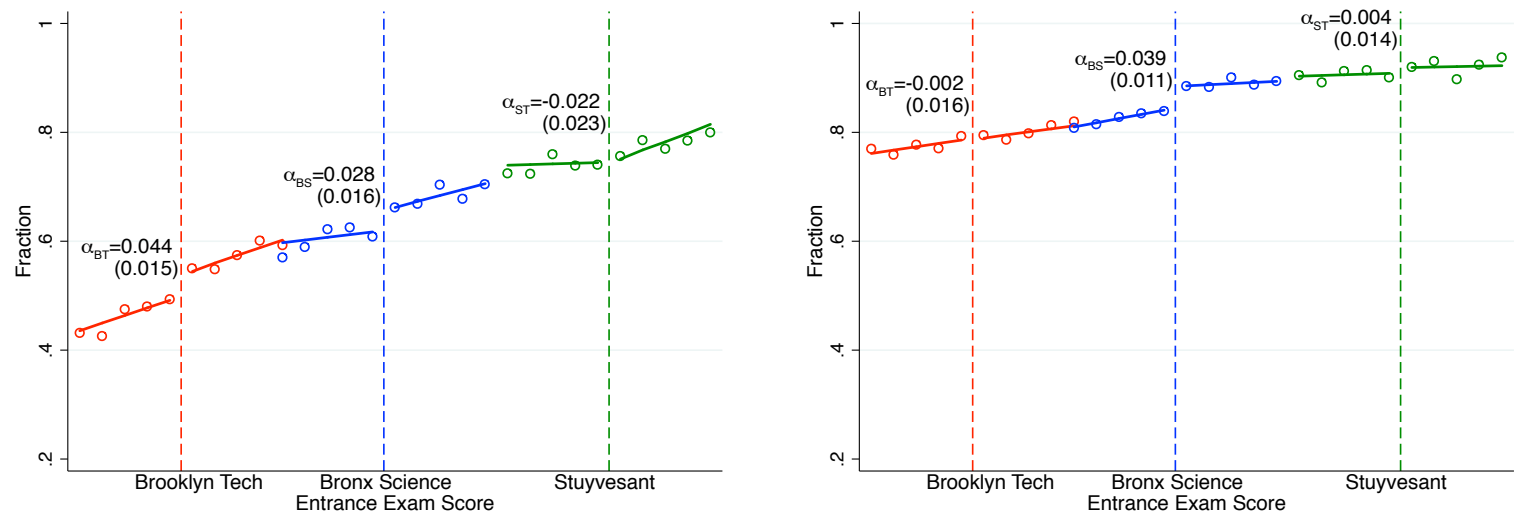

Notes: These figures plot exam school eligibility and high school graduation. The sample includes exam school applicants in the 2002 - 2009 high school cohorts. The smoothed line in each figure comes from a single local linear regression of each outcome on entrance exam score, school eligibility, and school eligibility interacted with the entrance exam score. Point estimates and standard errors clustered at the exam score level from an analogous regression that also controls for cohort fixed effects are presented next to each eligibility cutoff. The dependent variable for each regression is an indicator equal to one if the student graduates from a NYC public high school with the indicated diploma. See text for additional details. 
Appendix Figure 8

Exam School Eligibility and Regents Completion

Appx. Figure 8A: Global History

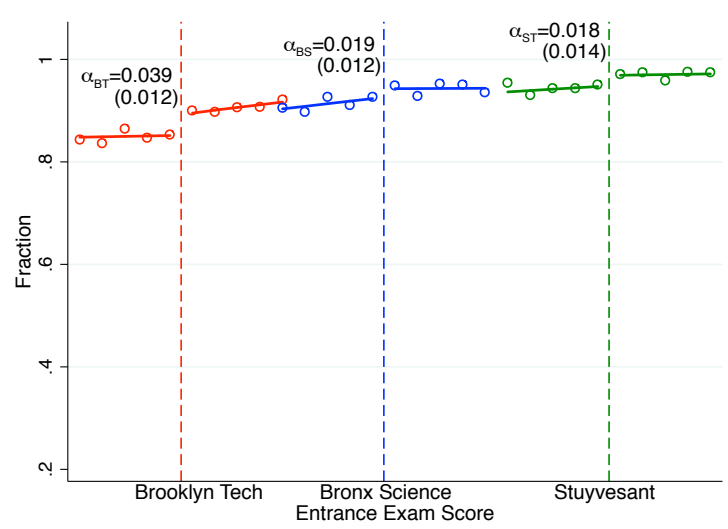

Appx. Figure 8C: ELA

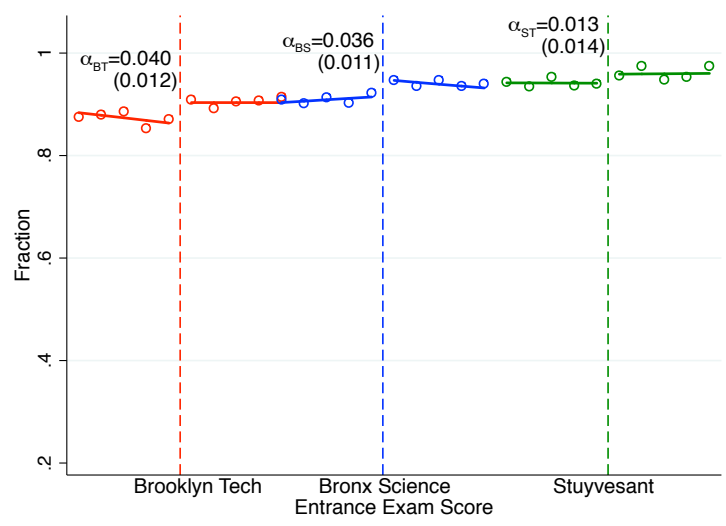

Appx. Figure 8E: Math 2

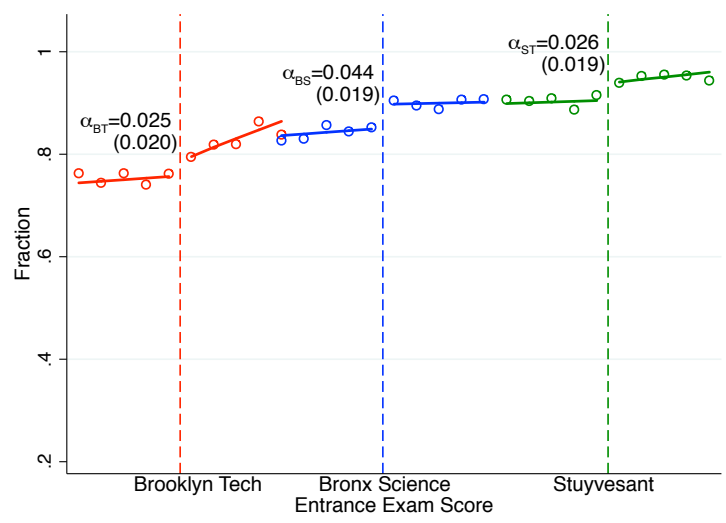

Appx. Figure 8B: U.S. History

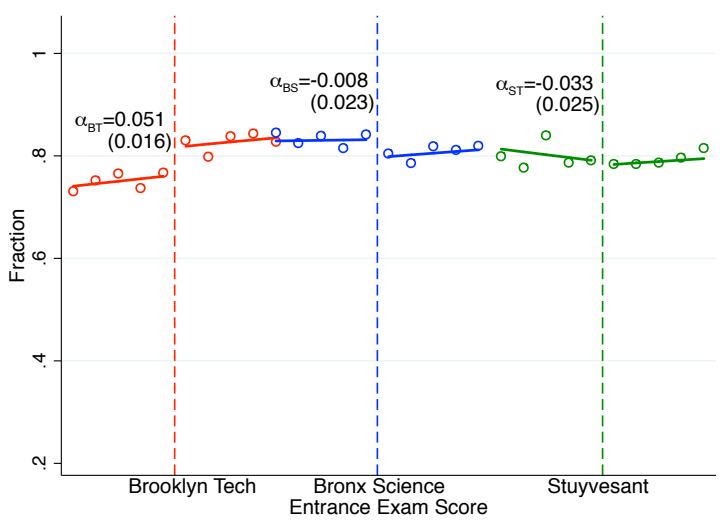

Appx. Figure 8D: Math 1

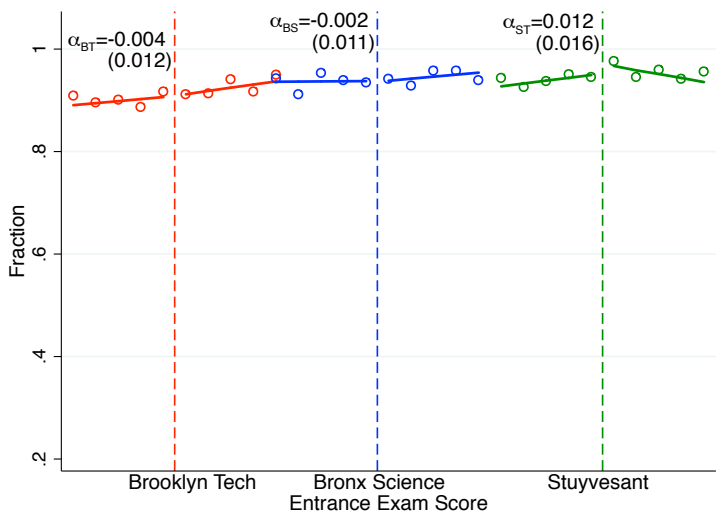

Appx. Figure 8F: Number of Science Exams

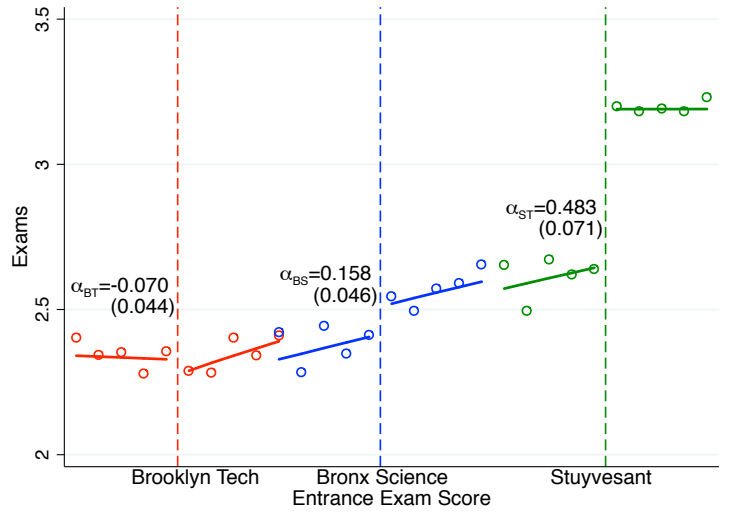


Appx. Figure 8G: Earth Systems Science

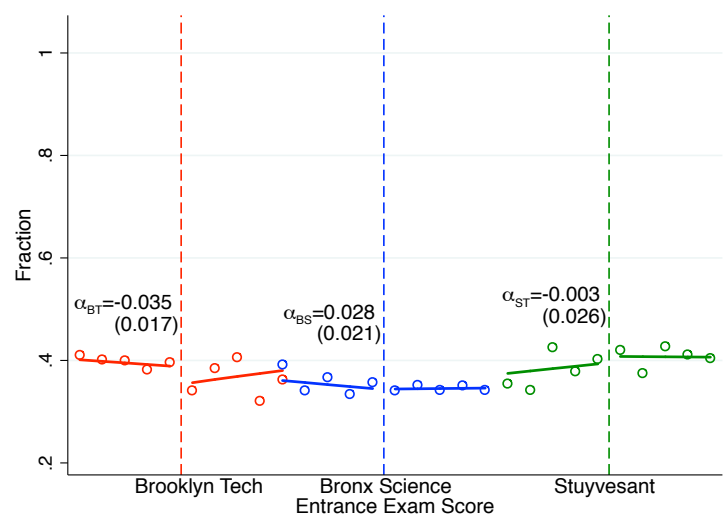

Appx. Figure 8I: Chemistry

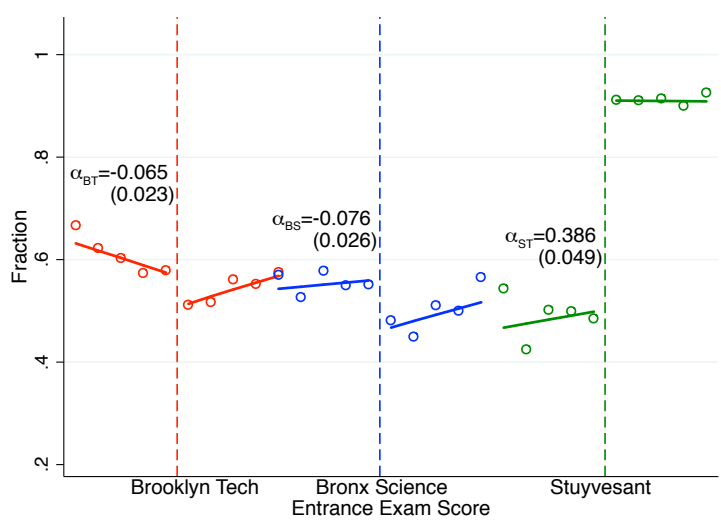

Appx. Figure 8H: Living Environment

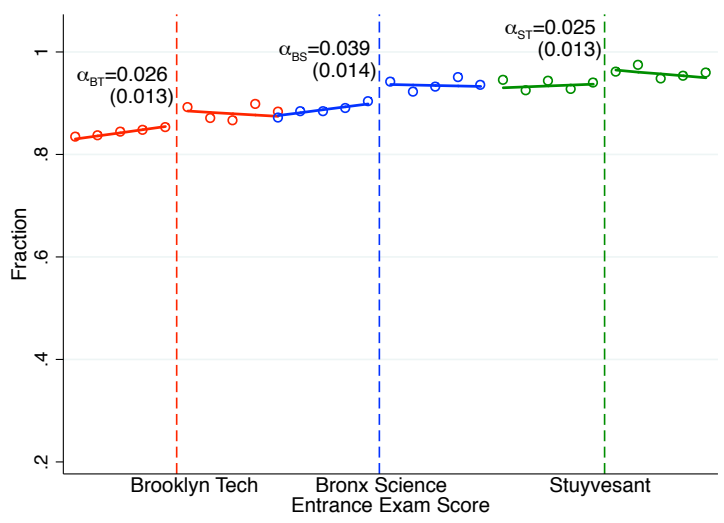

Appx. Figure 8J: Physics

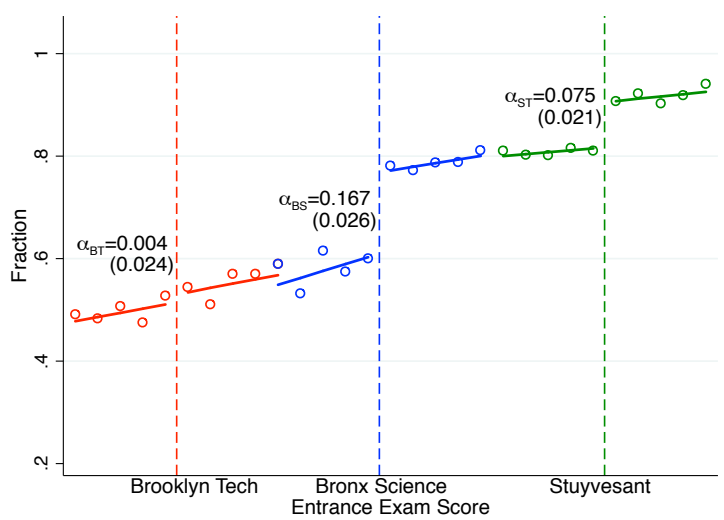

Notes: These figures plot exam school eligibility and high school Regents exam taking. The sample includes exam school applicants in the 2005 - 2009 high school cohorts. The smoothed line in each figure comes from a single local linear regression of each outcome on entrance exam score, school eligibility, and school eligibility interacted with the entrance exam score. Point estimates and standard errors clustered at the exam score level from an analogous regression that also controls for cohort fixed effects are presented next to each eligibility cutoff. The dependent variable for each regression is an indicator equal to one if the student passed the indicated Regents exam. See text for additional details. 


\section{Appendix Figure 9 \\ Exam School Eligibility and SAT Outcomes}

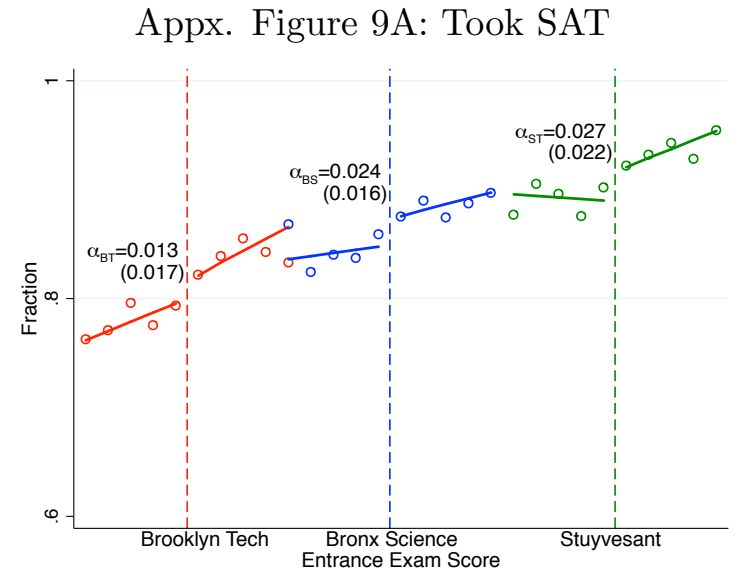

Appx. Figure 9C: SAT Reading Score

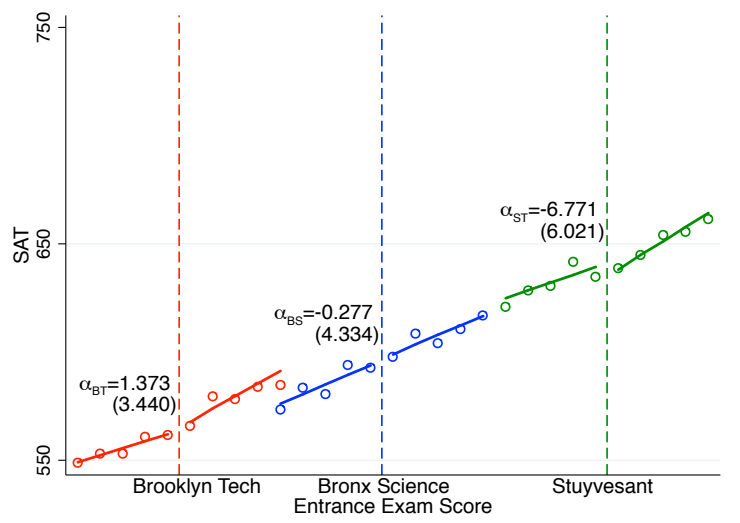

Appx. Figure 9B: SAT Combined Score

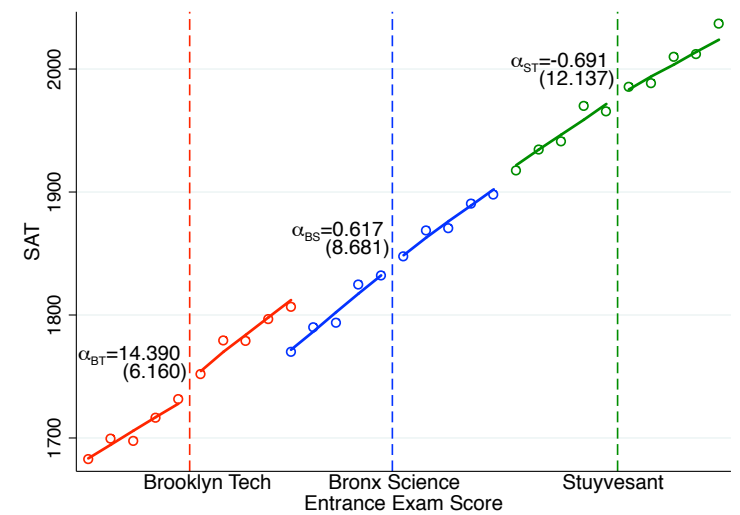

Appx. Figure 9D: SAT Writing Score

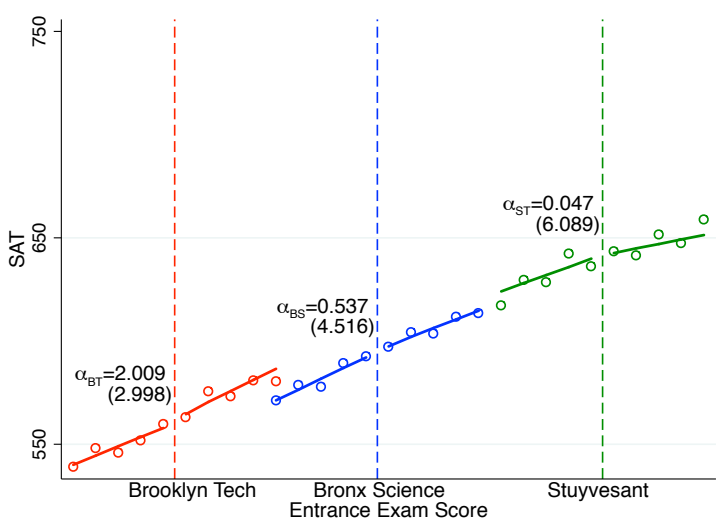

Figure 9E: SAT Math Score

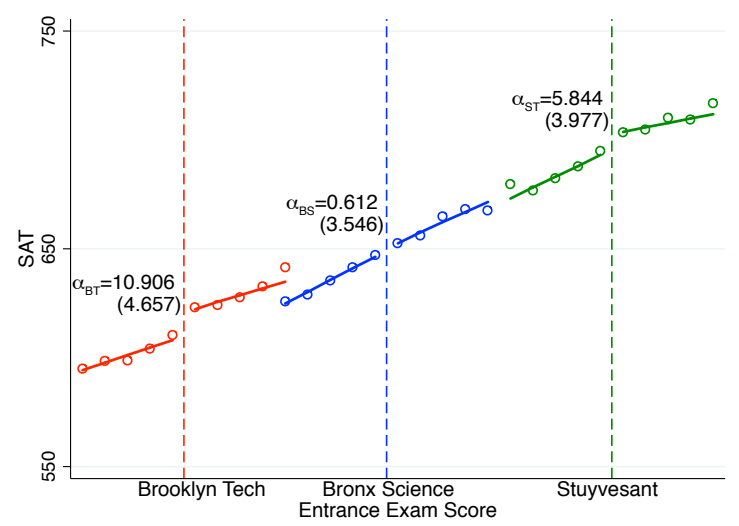

Notes: These figures plot exam school eligibility and SAT taking and SAT scores conditional on taking it. The sample includes exam school applicants in the 2007 - 2010 high school cohorts. The smoothed line in each figure comes from a single local linear regression of each outcome on entrance exam score, school eligibility, and school eligibility interacted with the entrance exam score. Point estimates and standard errors clustered at the exam score level from an analogous regression that also controls for cohort fixed effects are presented next to each eligibility cutoff. See text for additional details. 
Appendix Table 1

Admissions Cutoffs for NYC Exam Schools

\begin{tabular}{|c|c|c|c|c|c|c|c|}
\hline \multirow{3}{*}{$\begin{array}{l}\text { Grad } \\
\text { Cohort }\end{array}$} & \multirow[b]{2}{*}{ Exam } & \multicolumn{2}{|c|}{ Stuyvsant } & \multicolumn{2}{|c|}{ Bronx Science } & \multicolumn{2}{|c|}{ Brooklyn Tech } \\
\hline & & Rank & Score & Rank & Score & Rank & Score \\
\hline & Takers & Cutoff & Cutoff & Cutoff & Cutoff & Cutoff & Cutoff \\
\hline$(1)$ & $(2)$ & $(3)$ & $(4)$ & $(5)$ & $(6)$ & $(7)$ & $(8)$ \\
\hline 1994 & 14173 & 728 & 1.691 & 1995 & 1.104 & 5305 & 0.288 \\
\hline 1995 & 13963 & 683 & 1.712 & 1821 & 1.171 & 4444 & 0.454 \\
\hline 1996 & 14481 & 688 & 1.735 & 2118 & 1.100 & 4658 & 0.443 \\
\hline 1997 & 16087 & 766 & 1.739 & 2321 & 1.123 & 5040 & 0.452 \\
\hline 1998 & 16907 & 710 & 1.734 & 2114 & 1.183 & 4745 & 0.578 \\
\hline 1999 & 17188 & 817 & 1.725 & 2006 & 1.228 & 5021 & 0.537 \\
\hline 2000 & 17618 & 933 & 1.673 & 2248 & 1.177 & 4738 & 0.604 \\
\hline 2001 & 18280 & 888 & 1.741 & 2330 & 1.186 & 4092 & 0.773 \\
\hline 2002 & 18516 & 813 & 1.730 & 2245 & 1.170 & 4043 & 0.764 \\
\hline 2003 & 19202 & 865 & 1.744 & 2264 & 1.230 & 4088 & 0.814 \\
\hline 2004 & 19763 & 880 & 1.728 & 2202 & 1.241 & 3905 & 0.862 \\
\hline 2005 & 19921 & 958 & 1.727 & 2190 & 1.279 & 3701 & 0.929 \\
\hline 2006 & 20679 & 957 & 1.726 & 2075 & 1.312 & 3567 & 0.963 \\
\hline 2007 & 25037 & 866 & 1.871 & 2046 & 1.434 & 3211 & 1.172 \\
\hline 2008 & 26322 & 876 & 1.939 & 2313 & 1.410 & 3974 & 1.057 \\
\hline 2009 & 26619 & 1088 & 1.863 & 2641 & 1.362 & 4182 & 1.040 \\
\hline 2010 & 26707 & 1175 & 1.780 & 2966 & 1.265 & 4788 & 0.936 \\
\hline 2011 & 25080 & 1220 & 1.740 & 3108 & 1.213 & 4853 & 0.894 \\
\hline 2012 & 25871 & 1190 & 1.774 & 3289 & 1.182 & 4921 & 0.897 \\
\hline 2013 & 27650 & 1179 & 1.814 & 3046 & 1.284 & 4976 & 0.941 \\
\hline
\end{tabular}

Notes: This table reports the SHSAT cutoffs for each school and cohort. Test results are ranked from the highest score to the lowest, and administrators place students in high schools starting with the students with the highest score. Each student is placed into their most preferred school that still has seats until no seats remain at any exam school. We report the rank and standardized test score of the last student admitted to a school in each cohort. 
Appendix Table 2

Data Coverage by Cohort

\begin{tabular}{ccccccc}
\hline $\begin{array}{c}\text { Grad } \\
\text { Cohort }\end{array}$ & $\begin{array}{c}\text { Race } \\
\text { Data }\end{array}$ & $\begin{array}{c}\text { 8th State } \\
\text { Scores }\end{array}$ & $\begin{array}{c}\text { Regents } \\
\text { Scores }\end{array}$ & $\begin{array}{c}\text { SAT } \\
\text { Scores }\end{array}$ & $\begin{array}{c}\text { HS Grad } \\
\text { Data }\end{array}$ & $\begin{array}{c}\text { College } \\
\text { Enrollment }\end{array}$ \\
\hline$(1)$ & $(2)$ & $(3)$ & $(4)$ & $(5)$ & $(6)$ & $(7)$ \\
\hline 1994 & No & No & No & No & No & Yes \\
1995 & No & No & No & No & No & Yes \\
1996 & No & No & No & No & No & Yes \\
1997 & No & No & No & No & No & Yes \\
1998 & No & No & No & No & No & Yes \\
1999 & No & No & No & No & No & Yes \\
2000 & No & No & No & No & No & Yes \\
2001 & No & No & No & No & No & Yes \\
2002 & No & Yes & No & No & Yes & Yes \\
2003 & No & Yes & No & No & Yes & Yes \\
2004 & No & Yes & No & No & Yes & Yes \\
2005 & No & Yes & Yes & No & Yes & Yes \\
2006 & No & Yes & Yes & No & Yes & Yes \\
2007 & No & Yes & Yes & Yes & Yes & Yes \\
2008 & Yes & Yes & Yes & Yes & Yes & Yes \\
2009 & Yes & Yes & Yes & Yes & Yes & Yes \\
2010 & Yes & Yes & No & Yes & No & No \\
2011 & Yes & Yes & No & No & No & No \\
2012 & Yes & Yes & No & No & No & No \\
2013 & Yes & Yes & No & No & No & No \\
\hline \hline
\end{tabular}

Notes: This table reports the available data for each graduating high school cohort. 
Appendix Table 3

Exam School Eligibility and Graduation for All Schools

\begin{tabular}{lccccc}
\hline \hline & & Bronx & Q. Sci/ & & Brooklyn \\
& Stuyvesant & Science & Lehman & MSE & Tech \\
\cline { 2 - 6 } & $(1)$ & $(2)$ & $(3)$ & $(4)$ & $(5)$ \\
\cline { 2 - 6 } Stuyvesant & $0.695^{* * *}$ & $-0.336^{* * *}$ & -0.006 & -0.001 & $-0.157^{* * *}$ \\
& $(0.025)$ & $(0.029)$ & $(0.009)$ & $(0.005)$ & $(0.023)$ \\
Bronx Science & 3004 & 3004 & 3004 & 3004 & 3004 \\
& 0.001 & $0.346^{* * *}$ & $-0.074^{* * *}$ & $-0.021^{* *}$ & $-0.075^{* *}$ \\
Queens Science/Lehman & $(0.002)$ & $(0.021)$ & $(0.014)$ & $(0.009)$ & $(0.030)$ \\
& 5872 & 5872 & 5872 & 5872 & 5872 \\
MSE & $-0.005^{*}$ & $-0.006^{*}$ & $0.082^{* * *}$ & $-0.031^{* *}$ & $-0.060^{* *}$ \\
& $(0.003)$ & $(0.004)$ & $(0.011)$ & $(0.015)$ & $(0.029)$ \\
Brooklyn Tech & 7563 & 7563 & 7563 & 7563 & 7563 \\
& -0.007 & $-0.012^{* *}$ & $-0.020^{* *}$ & $0.068^{* * *}$ & $0.128^{* * *}$ \\
& $(0.008)$ & $(0.005)$ & $(0.010)$ & $(0.018)$ & $(0.041)$ \\
& 8046 & 8046 & 8046 & 8046 & 8046 \\
& -0.009 & $-0.015^{* * *}$ & $-0.026^{* * *}$ & 0.021 & $0.242^{* * *}$ \\
& $(0.011)$ & $(0.005)$ & $(0.009)$ & $(0.019)$ & $(0.030)$ \\
& 8150 & 8150 & 8150 & 8150 & 8150 \\
\hline \hline
\end{tabular}

Notes: This table reports reduced form estimates of the impact of exam school eligibility on high school graduation for all exam schools. The sample includes exam school applicants in the 2007 - 2009 high school cohorts. Each row reports the coefficient on exam school eligibility at the indicated school. The dependent variable for each regression is an indicator for graduating from the school listed at the top of each column. All specifications control for entrance exam score, entrance exam score interacted with school eligibility, and cohort fixed effects. Standard errors are clustered at the exam score level. The Queens Science and Lehman results are combined as the cutoffs overlap in most years. When not overlapping, we use the lower of the two cutoffs. $* * *=$ significant at 1 percent level, $* *=$ significant at 5 percent level, ${ }^{*}=$ significant at 10 percent level. 


\begin{tabular}{ccccc}
\multicolumn{5}{c}{ Appendix Table 4} \\
Exam School Eligibility and College Outcomes for All Schools \\
\hline \hline \multirow{5}{*}{ Stuyvesant } & Start & $1200+$ & $1300+$ & $1400+$ \\
& 4 -year & SAT & SAT & SAT \\
\cline { 2 - 5 } & $(1)$ & $(2)$ & $(3)$ & $(4)$ \\
\cline { 2 - 5 } Bronx Science & -0.012 & 0.029 & 0.029 & 0.039 \\
& $(0.029)$ & $(0.036)$ & $(0.039)$ & $(0.033)$ \\
& 2720 & 2720 & 2720 & 2720 \\
Queens Science/Lehman & 0.005 & 0.012 & 0.000 & -0.023 \\
& $(0.024)$ & $(0.023)$ & $(0.020)$ & $(0.018)$ \\
MSE & 5111 & 5111 & 5111 & 5111 \\
& $(0.007$ & -0.015 & -0.012 & 0.012 \\
Brooklyn Tech & 6415 & $6.023)$ & $(0.019)$ & $(0.012)$ \\
& 0.019 & -0.010 & -0.019 & $-0.029^{* *}$ \\
& $(0.027)$ & $(0.021)$ & $(0.018)$ & $(0.012)$ \\
& 6778 & 6778 & 6778 & 6778 \\
& 0.003 & -0.021 & -0.024 & $-0.029^{* *}$ \\
& $(0.025)$ & $(0.019)$ & $(0.019)$ & $(0.011)$ \\
& 6855 & 6855 & 6855 & 6855 \\
\hline \hline
\end{tabular}

Notes: This table reports reduced form estimates of the impact of exam school eligibility on college outcomes for all exam schools. The sample includes exam school applicants in the $2007-2009$ high school cohorts. Each row reports the coefficient on exam school eligibility at the indicated school. The dependent variable for each regression is indicated at the top of each column. All specifications control for entrance exam score, entrance exam score interacted with school eligibility, and cohort fixed effects. Standard errors are clustered at the exam score level. The Queens Science and Lehman results are combined as the cutoffs overlap in most years. When not overlapping, we use the lower of the two cutoffs. ${ }^{* * *}=$ significant at 1 percent level, $* *=$ significant at 5 percent level, ${ }^{*}=$ significant at 10 percent level. 


\begin{tabular}{ccccc}
\multicolumn{5}{c}{ Appendix Table 5 } \\
Exam School Eligibility \\
and High School Outcomes for All Schools \\
\hline \hline \multirow{5}{*}{ Stuyvesant } & Grad & Regents & Advanced & Either \\
& HS & Diploma & Regents & Regents \\
\cline { 2 - 5 } & $(1)$ & $(2)$ & $(3)$ & $(4)$ \\
\cline { 2 - 5 } Bronx Science & -0.008 & -0.007 & -0.006 & -0.013 \\
& $(0.015)$ & $(0.019)$ & $(0.022)$ & $(0.017)$ \\
Queens Science/Lehman & 2251 & 2251 & 2251 & 2251 \\
& $0.057^{* * *}$ & $-0.031^{*}$ & $0.079^{* * *}$ & $0.048^{* * *}$ \\
& $(0.014)$ & $(0.017)$ & $(0.024)$ & $(0.013)$ \\
MSE & 4319 & 4319 & 4319 & 4319 \\
& 0.021 & -0.015 & 0.036 & 0.021 \\
Brooklyn Tech & $0.019)$ & $(0.016)$ & $(0.023)$ & $(0.019)$ \\
& 5488 & 5488 & 5488 & 5488 \\
& $0.031^{*}$ & -0.009 & 0.025 & 0.016 \\
& $(0.017)$ & $(0.024)$ & $(0.028)$ & $(0.019)$ \\
& 5789 & 5789 & 5789 & 5789 \\
& $0.029^{*}$ & -0.029 & $0.054^{*}$ & 0.025 \\
& $(0.017)$ & $(0.026)$ & $(0.028)$ & $(0.017)$ \\
& 5866 & 5866 & 5866 & 5866 \\
\hline \hline
\end{tabular}

Notes: This table reports reduced form estimates of the impact of exam school eligibility on high school outcomes for all exam schools. The sample includes exam school applicants in the $2007-2009$ high school cohorts. Each row reports the coefficient on exam school eligibility at the indicated school. The dependent variable for each regression is indicated at the top of each column. All specifications control for entrance exam score, entrance exam score interacted with school eligibility, and cohort fixed effects. Standard errors are clustered at the exam score level. The Queens Science and Lehman results are combined as the cutoffs overlap in most years. When not overlapping, we use the lower of the two cutoffs. ${ }^{* * *}=$ significant at 1 percent level, $* *=$ significant at 5 percent level, ${ }^{*}=$ significant at 10 percent level. 


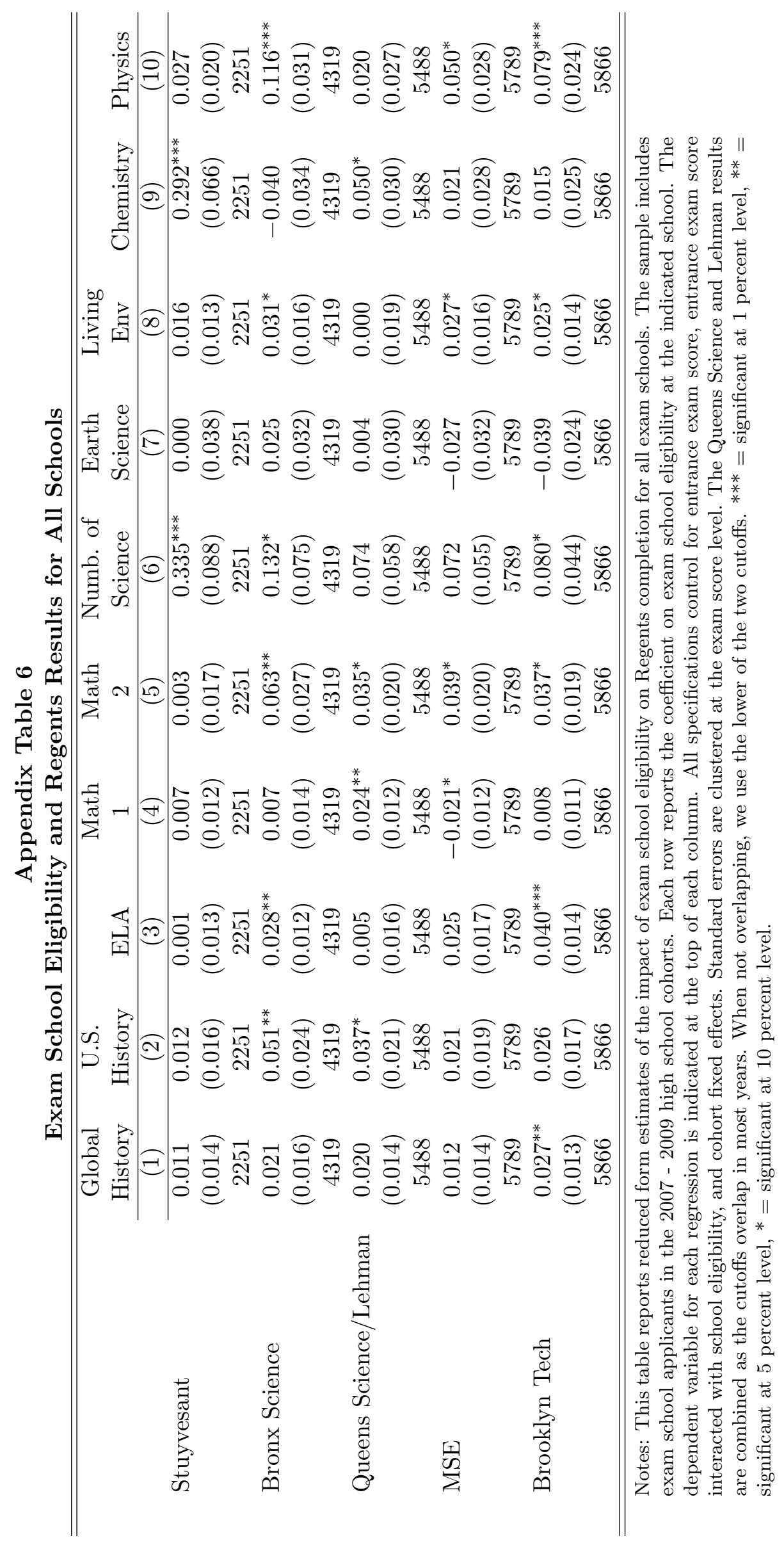




\begin{tabular}{ccc}
\multicolumn{3}{c}{ Appendix Table 7} \\
Exam School Eligibility and SAT Results & for All Sch \\
\hline \hline & Took & SAT \\
& SAT & Score \\
\cline { 2 - 3 } Stuyvesant & $(1)$ & $(2)$ \\
\cline { 2 - 3 } & 0.023 & -6.271 \\
Bronx Science & $0.030)$ & $(22.850)$ \\
& 3159 & 1015 \\
Queens Science/Lehman & 0.022 & 19.274 \\
& $(0.021)$ & $(12.618)$ \\
& 5996 & 1800 \\
MSE & 0.005 & 2.030 \\
& $(0.020)$ & $(12.703)$ \\
& 7544 & 2195 \\
Brooklyn Tech & 0.008 & 17.383 \\
& $(0.019)$ & $(13.259)$ \\
& 7998 & 2297 \\
& -0.003 & -3.021 \\
& $(0.016)$ & $(12.866)$ \\
& 8075 & 2320 \\
\hline
\end{tabular}

Notes: This table reports reduced form estimates of the impact of exam school eligibility on SAT outcomes for all exam schools. The sample includes exam school applicants in the $2007-2009$ high school cohorts. Each row reports the coefficient on exam school eligibility at the indicated school. The dependent variable for each regression is indicated at the top of each column. All specifications control for entrance exam score, entrance exam score interacted with school eligibility, and cohort fixed effects. Standard errors are clustered at the exam score level. The Queens Science and Lehman results are combined as the cutoffs overlap in most years. When not overlapping, we use the lower of the two cutoffs. ${ }^{* * *}=$ significant at 1 percent level, ${ }^{* *}=$ significant at 5 percent level, ${ }^{*}=$ significant at 10 percent level. 
Appendix Table 8

Exam School Eligibility and College Outcomes for NYC Matched Sample

\begin{tabular}{ccccccc}
\hline \hline & Start & Grad & $1200+$ & $1300+$ & $1400+$ & Post \\
& 4 -year & College & SAT & SAT & SAT & Grad \\
\cline { 2 - 7 } Stuyvesant & $(1)$ & $(2)$ & $(3)$ & $(4)$ & $(5)$ & $(6)$ \\
\cline { 2 - 7 } & -0.003 & 0.043 & $0.071^{* *}$ & 0.042 & $0.036^{*}$ & 0.013 \\
& $(0.021)$ & $(0.047)$ & $(0.028)$ & $(0.028)$ & $(0.019)$ & $(0.036)$ \\
Bronx Science & 5569 & 1991 & 5569 & 5569 & 5569 & 1991 \\
& -0.010 & -0.007 & 0.005 & -0.015 & $-0.022^{* *}$ & $-0.048^{*}$ \\
Brooklyn Tech & $(0.015)$ & $(0.038)$ & $(0.018)$ & $(0.018)$ & $(0.011)$ & $(0.026)$ \\
& 10504 & 4026 & 10504 & 10504 & 10504 & 4026 \\
& -0.001 & -0.044 & 0.001 & -0.002 & -0.006 & -0.018 \\
& $(0.016)$ & $(0.030)$ & $(0.013)$ & $(0.010)$ & $(0.006)$ & $(0.022)$ \\
\hline \hline
\end{tabular}

Notes: This table reports reduced form estimates of the impact of exam school eligibility on college outcomes. The sample is restricted to exam school applicants in the 2002 - 2009 high school cohorts who attended a public NYC middle school. Each row reports the coefficient on exam school eligibility at the indicated school. The dependent variable for each regression is indicated at the top of each column. All specifications control for entrance exam score, entrance exam score interacted with school eligibility, and cohort fixed effects. Standard errors are clustered at the exam score level. The Queens Science and Lehman results are combined as the cutoffs overlap in most years. When not overlapping, we use the lower of the two cutoffs. ${ }^{* * *}=$ significant at 1 percent level, ${ }^{* *}=$ significant at 5 percent level, ${ }^{*}=$ significant at 10 percent level. 
Appendix Table 9

Robustness of Main Outcomes

\begin{tabular}{|c|c|c|c|c|c|c|}
\hline Polynomial Order & 1 & 1 & 1 & 2 & 2 & 2 \\
\hline \multirow[t]{2}{*}{ Bandwidth } & 0.15 & 0.25 & 0.35 & 0.15 & 0.25 & 0.35 \\
\hline & $(1)$ & $(2)$ & $(3)$ & $(4)$ & $(5)$ & $(6)$ \\
\hline \multicolumn{7}{|c|}{ Panel A: Start 4 year College } \\
\hline \multirow[t]{2}{*}{ Stuyvesant } & $\begin{array}{r}-0.019 \\
(0.017)\end{array}$ & $\begin{array}{r}-0.013 \\
(0.014)\end{array}$ & $\begin{array}{r}-0.015 \\
(0.012)\end{array}$ & $\begin{array}{r}-0.032 \\
(0.028)\end{array}$ & $\begin{array}{r}-0.018 \\
(0.019)\end{array}$ & $\begin{array}{r}-0.014 \\
(0.017)\end{array}$ \\
\hline & 8313 & 14156 & 20334 & 8313 & 14156 & 20334 \\
\hline \multirow[t]{3}{*}{ Bronx Science } & 0.005 & 0.002 & 0.003 & 0.013 & 0.006 & 0.003 \\
\hline & $(0.013)$ & $(0.010)$ & $(0.009)$ & $(0.019)$ & $(0.014)$ & $(0.012)$ \\
\hline & 16110 & 27120 & 38241 & 16110 & 27120 & 38241 \\
\hline \multirow[t]{3}{*}{ Brookyln Tech } & 0.002 & -0.008 & -0.010 & 0.016 & 0.004 & -0.006 \\
\hline & $(0.012)$ & $(0.009)$ & $(0.008)$ & $(0.018)$ & $(0.013)$ & $(0.011)$ \\
\hline & 23059 & 39007 & 54879 & 23059 & 39007 & 54879 \\
\hline \multicolumn{7}{|c|}{ Panel B: Graduate from 4 year College } \\
\hline \multirow[t]{3}{*}{ Stuyvesant } & -0.027 & -0.016 & -0.008 & $-0.059^{*}$ & -0.028 & -0.027 \\
\hline & $(0.025)$ & $(0.021)$ & $(0.018)$ & $(0.035)$ & $(0.028)$ & $(0.025)$ \\
\hline & 5624 & 9558 & 13624 & 5624 & 9558 & 13624 \\
\hline \multirow[t]{3}{*}{ Bronx Science } & 0.012 & -0.007 & -0.008 & 0.028 & 0.021 & 0.001 \\
\hline & $(0.019)$ & $(0.018)$ & $(0.015)$ & $(0.022)$ & $(0.020)$ & $(0.020)$ \\
\hline & 11039 & 18709 & 26264 & 11039 & 18709 & 26264 \\
\hline \multirow[t]{3}{*}{ Brookyln Tech } & -0.013 & $-0.023^{*}$ & $-0.025^{* *}$ & 0.009 & -0.008 & -0.021 \\
\hline & $(0.015)$ & $(0.012)$ & $(0.010)$ & $(0.023)$ & $(0.017)$ & $(0.015)$ \\
\hline & 16372 & 27850 & 38895 & 16372 & 27850 & 38895 \\
\hline \multicolumn{7}{|c|}{ Panel C: Graduate with Adv. Regents Diploma } \\
\hline \multirow[t]{3}{*}{ Stuyvesant } & -0.042 & -0.022 & -0.022 & -0.044 & -0.042 & -0.026 \\
\hline & $(0.025)$ & $(0.023)$ & $(0.020)$ & $(0.033)$ & $(0.028)$ & $(0.027)$ \\
\hline & 3363 & 5734 & 8315 & 3363 & 5734 & 8315 \\
\hline \multirow[t]{3}{*}{ Bronx Science } & $0.035^{*}$ & $0.028^{*}$ & $0.023^{*}$ & 0.043 & $0.042^{*}$ & $0.034^{*}$ \\
\hline & $(0.021)$ & $(0.016)$ & $(0.014)$ & $(0.033)$ & $(0.025)$ & $(0.021)$ \\
\hline & 6501 & 10955 & 15510 & 6501 & 10955 & 15510 \\
\hline \multirow[t]{3}{*}{ Brookyln Tech } & $0.054^{* * *}$ & $0.044^{* * *}$ & $0.040^{* * *}$ & $0.045^{*}$ & $0.058^{* * *}$ & $0.051^{* * *}$ \\
\hline & $(0.018)$ & $(0.015)$ & $(0.013)$ & $(0.026)$ & $(0.021)$ & $(0.019)$ \\
\hline & 8678 & 14688 & 20989 & 8678 & 14688 & 20989 \\
\hline \multicolumn{7}{|l|}{ Panel D: SAT Score } \\
\hline \multirow[t]{3}{*}{ Stuyvesant } & 4.384 & -0.691 & -3.193 & 18.485 & 5.428 & 2.121 \\
\hline & $(13.555)$ & $(12.137)$ & $(10.754)$ & $(17.561)$ & $(15.017)$ & $(14.256)$ \\
\hline & 1714 & 2874 & 4150 & 1714 & 2874 & 4150 \\
\hline \multirow[t]{3}{*}{ Bronx Science } & -2.259 & 0.617 & 1.249 & -7.468 & -3.965 & -0.791 \\
\hline & $(10.230)$ & $(8.681)$ & $(7.650)$ & $(12.298)$ & $(11.529)$ & $(10.264)$ \\
\hline & 3113 & 5167 & 7228 & 3113 & 5167 & 7228 \\
\hline \multirow[t]{3}{*}{ Brookyln Tech } & 8.184 & $14.390^{* *}$ & $12.835^{* *}$ & 13.576 & 6.092 & $15.037^{* *}$ \\
\hline & $(7.036)$ & $(6.160)$ & $(5.532)$ & $(10.306)$ & $(8.249)$ & $(7.158)$ \\
\hline & 3839 & 6506 & 9150 & 3839 & 6506 & 9150 \\
\hline
\end{tabular}

Notes: This table reports reduced form estimates of the impact of exam school eligibility using a variety of bandwidths and polynomial choices. The sample is restricted to exam school applicants in the $2002-2009$ high school cohorts who attended a public NYC middle school. Each row reports the coefficient on exam school eligibility at the indicated school. All specifications control for entrance exam score and entrance exam score interacted with school eligibility with the indicated degree of polynomial, and cohort fixed effects. Standard errors are clustered at the exam score level. Additional outcomes are available on request. $* * *=$ significant at 1 percent level, ${ }^{* *}=$ significant at 5 percent level, ${ }^{*}=$ significant at 10 percent level. 


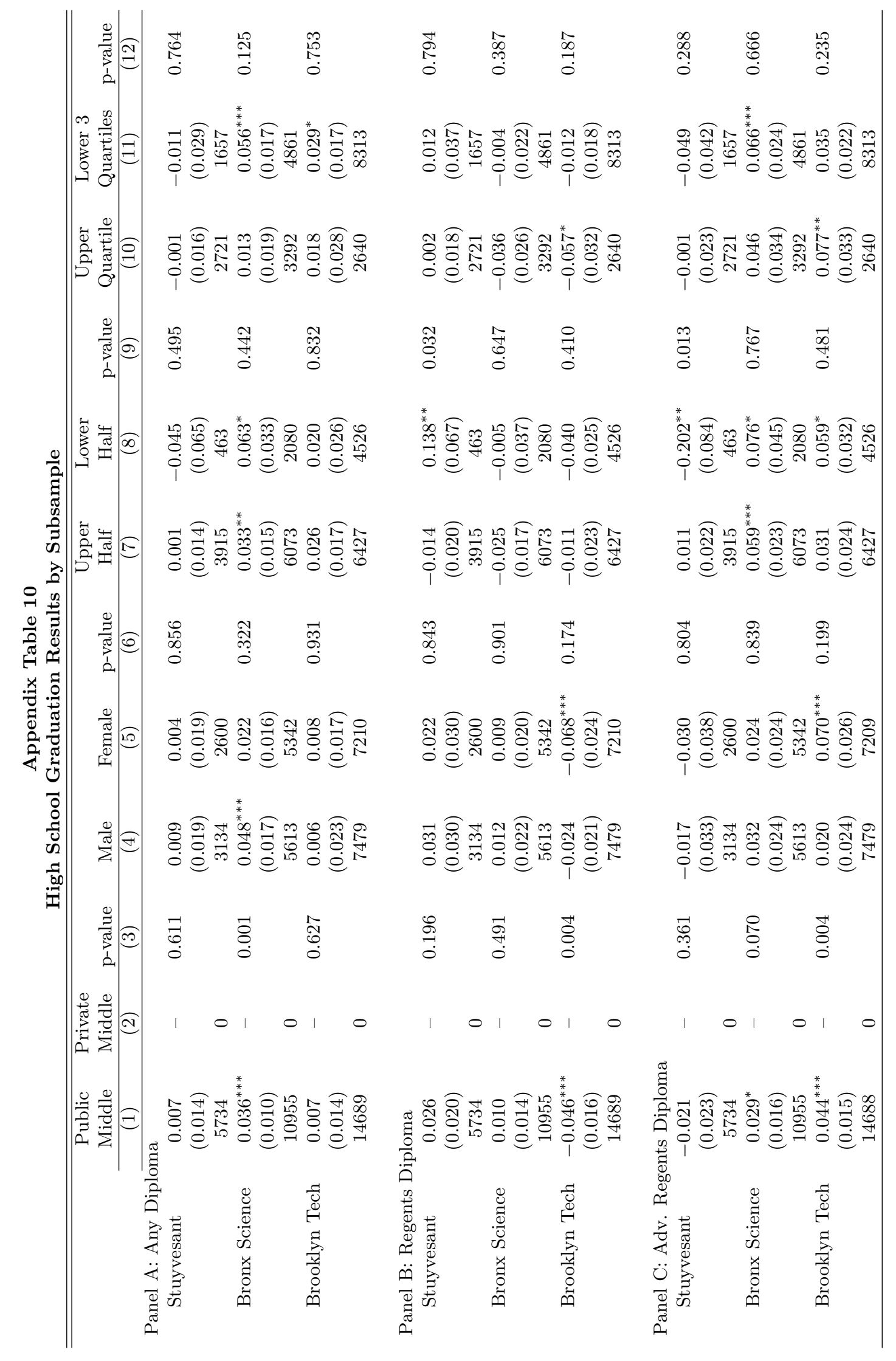




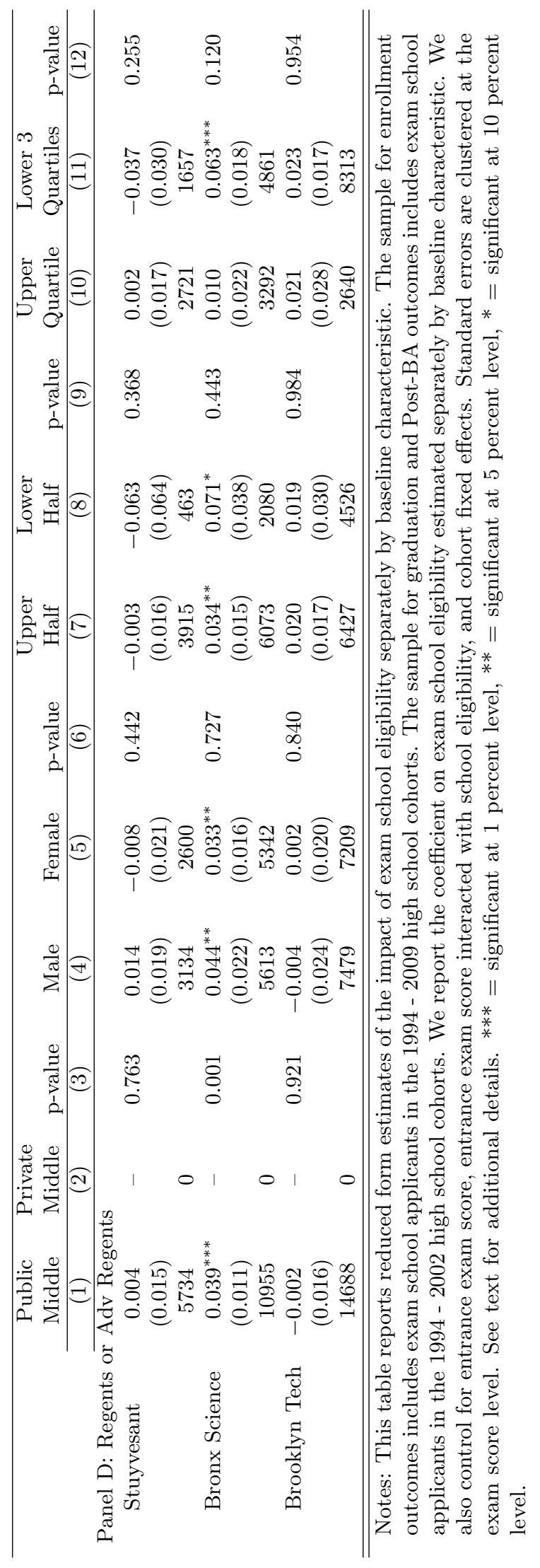




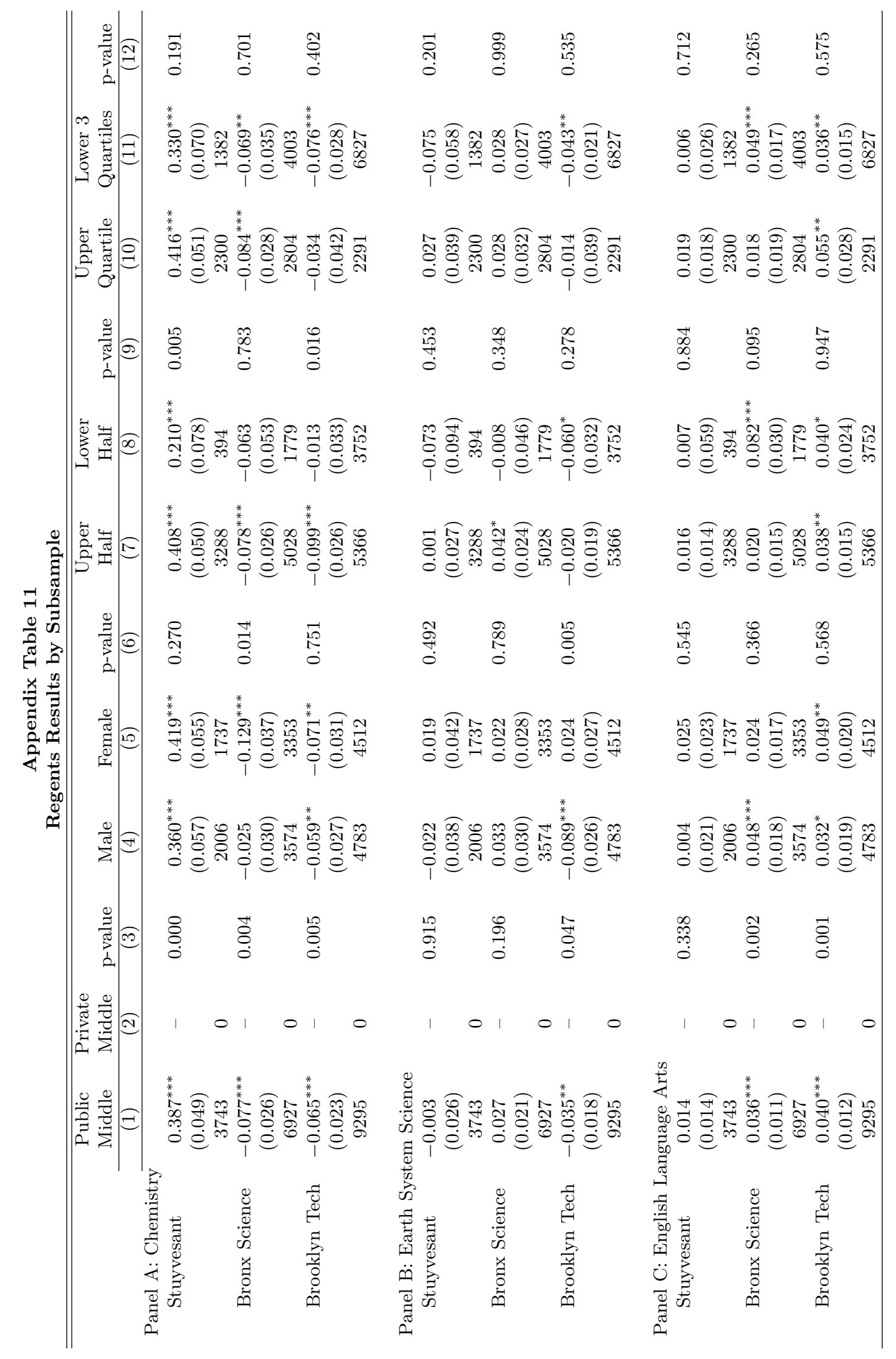




\begin{tabular}{|c|c|c|}
\hline \\
\hline 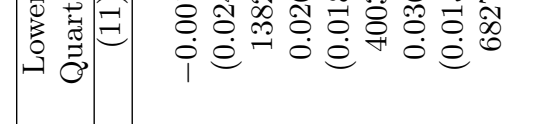 & 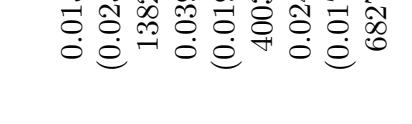 & 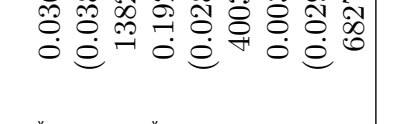 \\
\hline 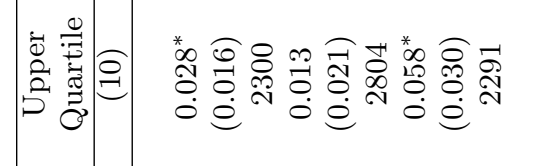 & 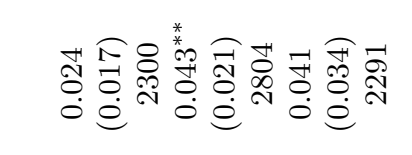 & 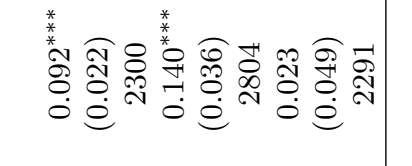 \\
\hline 淁 & 总 & 善 \\
\hline 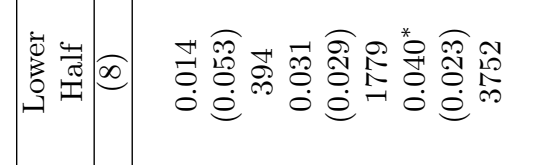 & 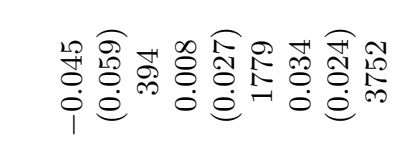 & \\
\hline 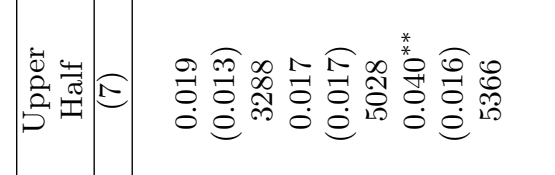 & 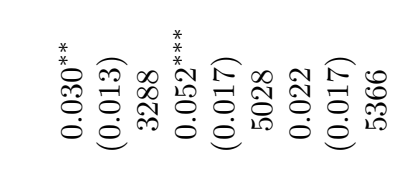 & 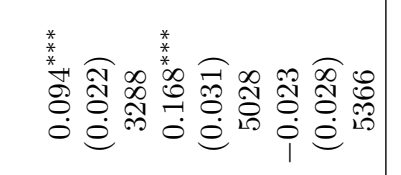 \\
\hline 器 & 㖕 & 善 亳 \\
\hline 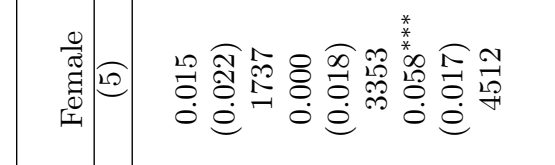 & 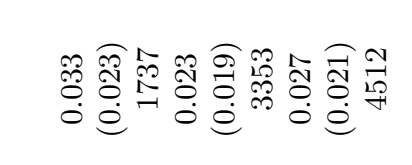 & 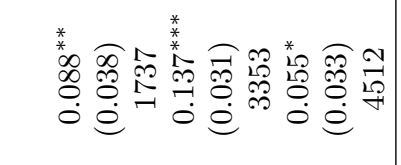 \\
\hline 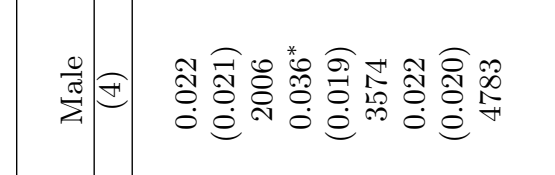 & 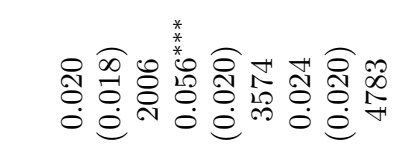 & 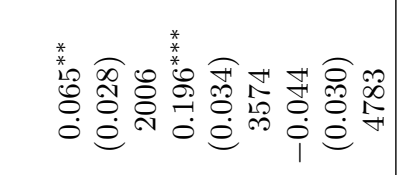 \\
\hline 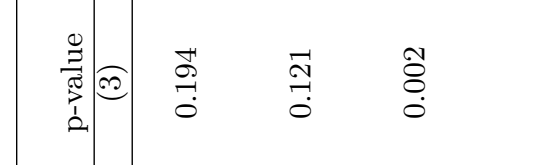 & 言器器 & 言 \\
\hline 101010 & & \\
\hline 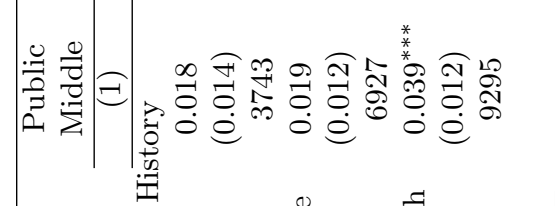 & 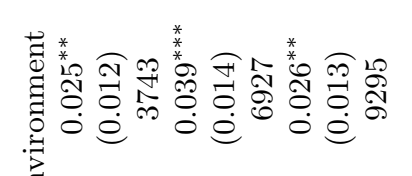 & \\
\hline 咅 & 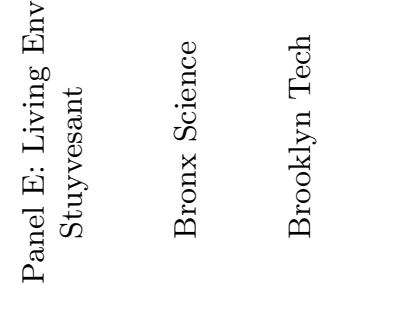 & 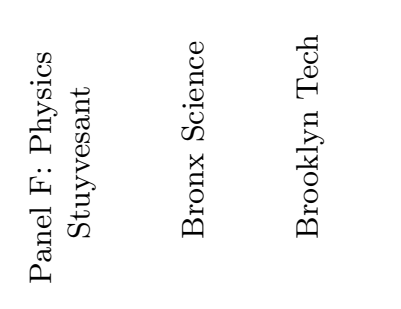 \\
\hline
\end{tabular}




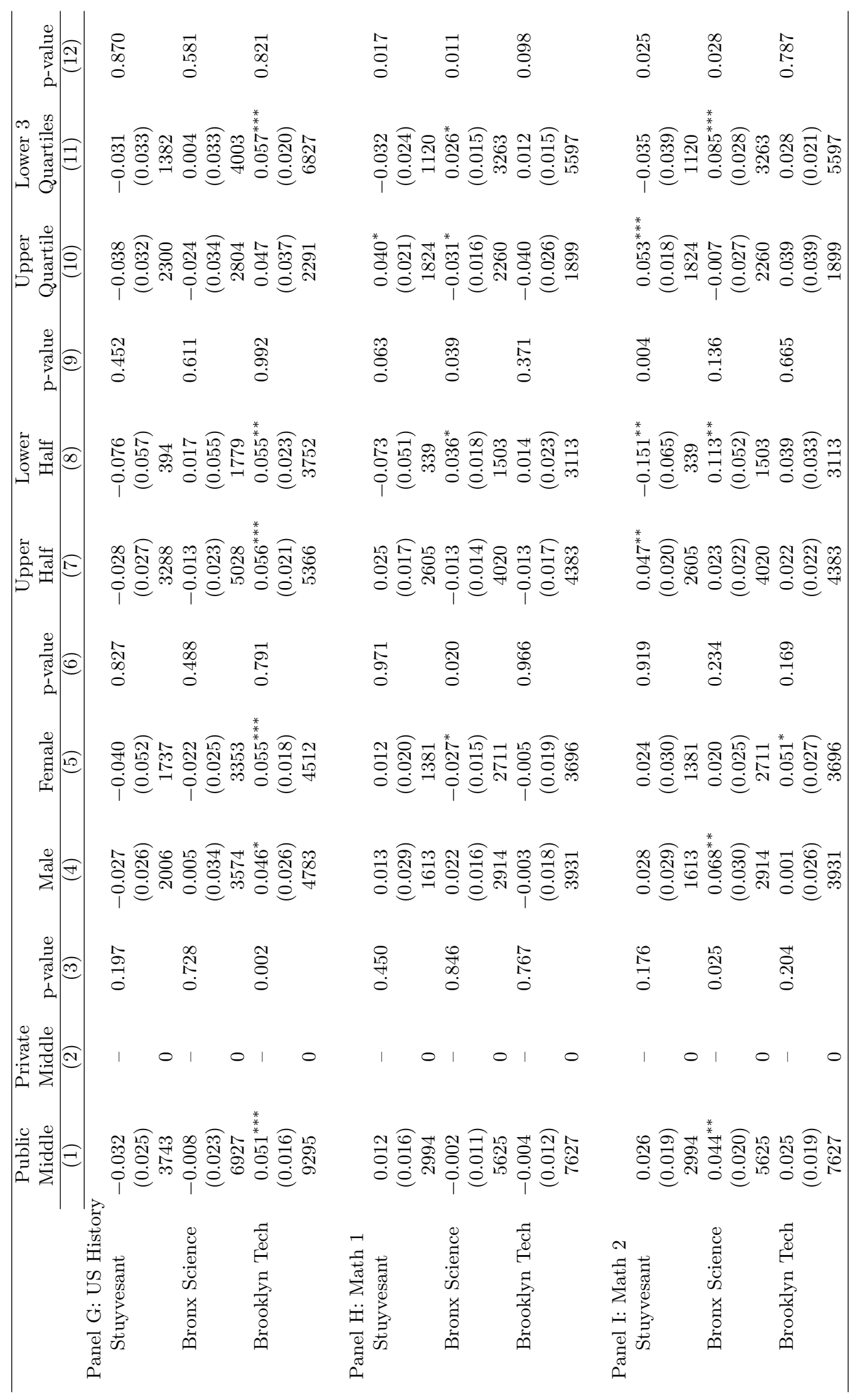




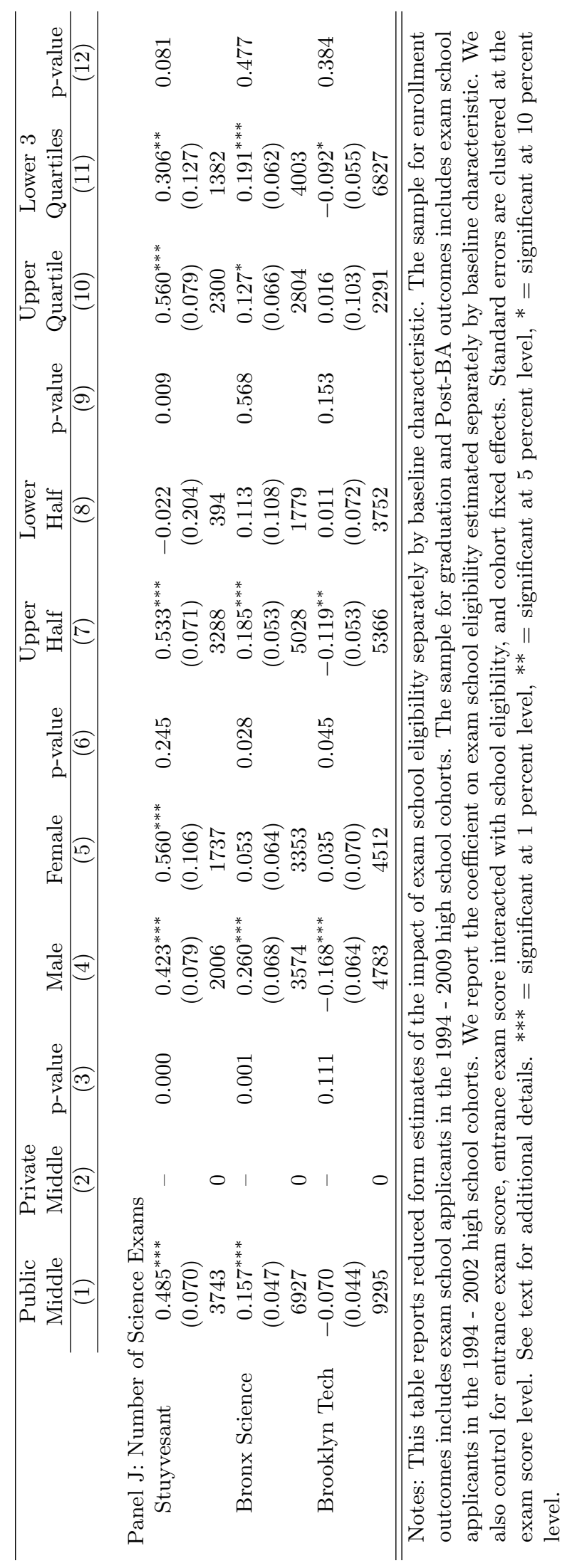




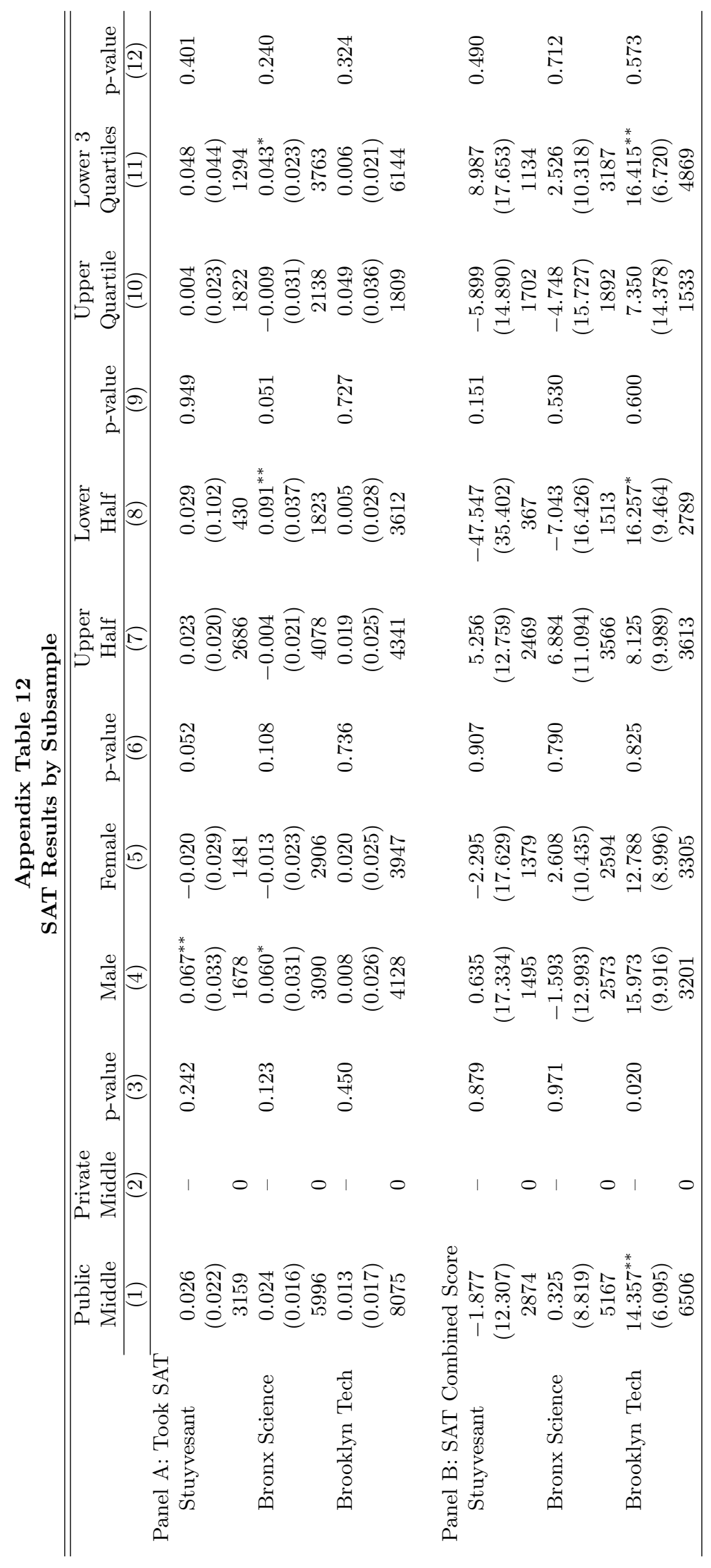




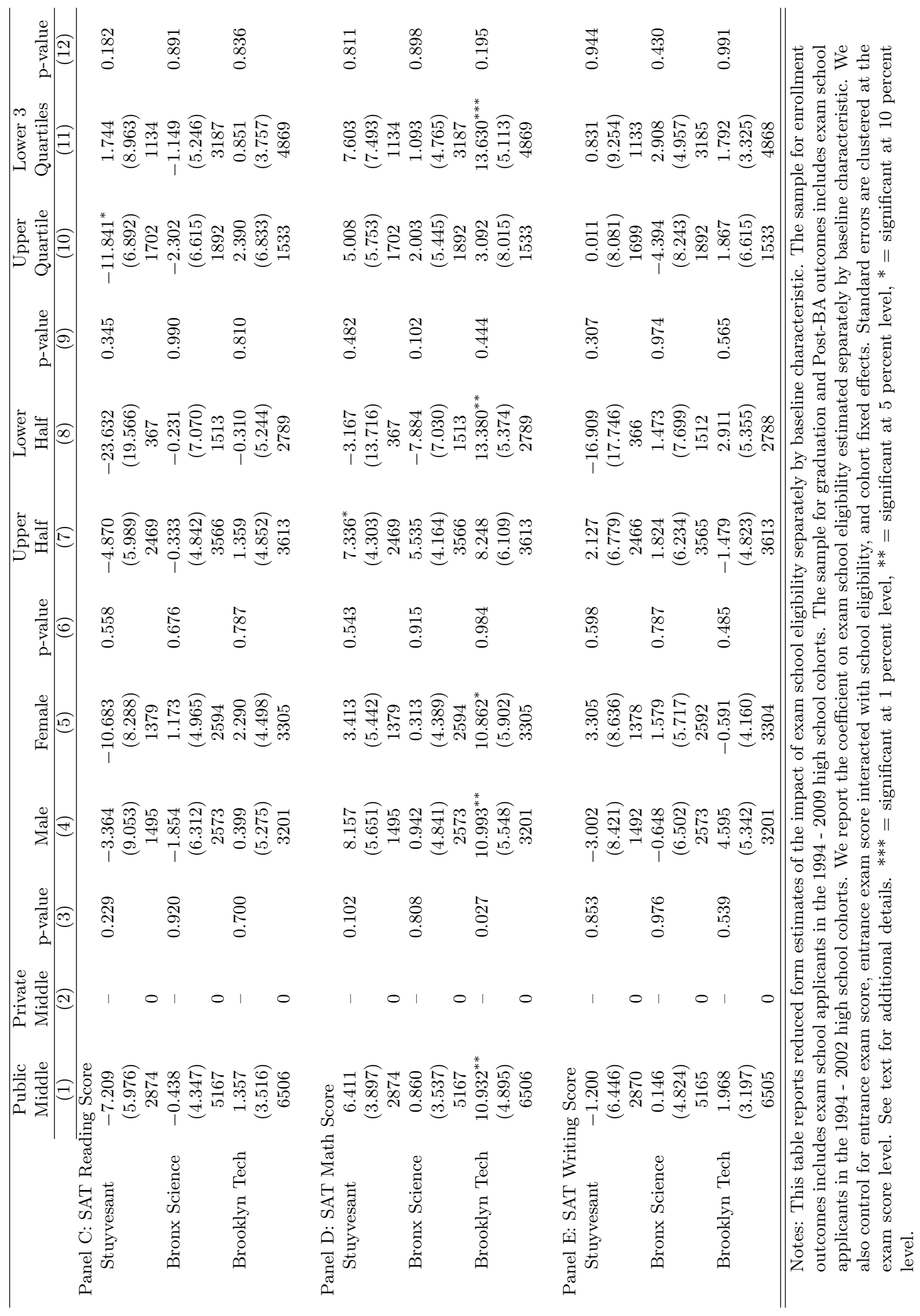




\section{Appendix B: Data Description and Construction of Variables}

\section{$1.1 \quad$ New York City Administrative Data}

\section{Demographic variables}

Demographic information was pulled from New York City enrollment files spanning the 200304 to 2009 -10 school years, with precedence given to the most recent file. Race consisted of the following categories: Black, Hispanic, White, Asian, and Other. These categories are considered mutually exclusive.

A student was considered free lunch if he was coded as "A" or "1" in the raw data, which corresponds to free lunch or "2" which corresponds to reduced-price lunch. A student was considered non free lunch if the student was coded as a "3", which corresponds to Full Price. All other values, including blanks, were coded as missing.

\section{New York State 8th Grade Test Scores}

State test scores in eighth grade were pulled from the NYC test score files spanning the 1999 - 2000 to 2009 - 2010 school years. Scores were standardized by year and grade to have mean of zero and standard deviation of one. The state mathematics and English Language Arts tests, developed by McGraw-Hill, are exams conducted in the winters of third through eighth grade. The math test includes questions on number sense and operations, algebra, geometry, measurement, and statistics. Tests in later grades focus on advanced topics such as algebra and geometry. The ELA test is designed to assess students on three learning standards - information and understanding, literary response and expression, critical analysis and evaluation - and includes multiple-choice and short-response sections based on a reading and listening section, along with a brief editing task.

\section{New York State Regents Test Scores}

Regents test scores for high school subjects were pulled from the NYC Regents test score files for 1998 - 1999 through 2009 - 2010. For each subject we construct indicator variables for a student having taken the exam, for having passed the exam at the basic level (55 out of 100), for having passed the exam at the Regents level (65 out of 100), and for having obtained mastery in the subject (85 out of 100). As the structure of the Math exams have changed over our sample period, we combine Sequential Math 1, Math A and Integrated Algebra scores and Sequential Math 3, Math B, and Trigonometry scores (based on the advice of NYC staff). Results are identical if we restrict the results to Math A and B, which make up the majority of our observations.

Regents exams are administered within schools in January, June, and August of each calendar year and are given in a wide variety of subjects, but scores range from 0 to 100 for every Regents exam. Students typically take exams at the end of the corresponding course, so that most students take the exams in June. Unlike most other standardized exams, teachers grade the Regents exams for students in their own school. The State Education Department of New York provides explicit guidelines for how the teacher-based scoring of each Regents exam should be organized.

Regents exam requirements have changed somewhat during the years we examine (1998 to 2010). To graduate, students generally must score at least 55 on each of five core Regents examinations: English, Mathematics, Science, U.S. History and Government, and Global History and Geography. In order to receive a more prestigious Regents Diploma, students must receive a score of at least 65 in each of these five core subjects. To earn an Advanced Regents Diploma, students must also score at least a 65 on elective exams in math, science, and foreign language.

Currently, the option of receiving a local diploma is being eliminated entirely. Beginning with those who entered the ninth grade in the fall of 2008, students are required to meet the Regents Diploma requirements (score 65 or higher in each of the five core subjects) in order to graduate 
from high school in New York State. The shift from local diploma to Regents diploma requirements was done gradually, with students entering ninth grade in fall 2005 having to score 65 in at least two core subjects, and each subsequent cohort facing stricter requirements.

A score of 85 is labeled as achieving mastery of the subject matter. While scoring 85 or higher is not relevant for high school graduation, meeting this cutoff is often used by high schools as a prerequisite for courses and by New York State colleges as either a prerequisite or qualification for credit towards a degree. Beginning with students who entered ninth grade in the fall of 2009, an additional accolade of Annotation of Mastery in science and/or math became available for students who score above 85 on three Regents exams in science and/or math.

Regents examinations contain both multiple-choice and open-response questions. The foreign language exams also contain a speaking component. Scoring materials provided to schools include the correct answers to multiple-choice questions and detailed, subject-specific instructions and procedures for evaluating open-ended and essay questions.

\section{School Characteristics}

School-level student variables were constructed for each school based on the population of students who were assigned to that high school in the NYC graduation files for the 2002 through 2009 high school cohorts.

School-level teacher variables were constructed for each school based on the 2008 - 2009 Human Resources file. We define teacher salaries as the mean salary for all school staff designated as full time teachers. Teacher experience is defined similarly. Student to teacher and staff ratios were constructed using 2008 - 2009 staffing levels and the average cohort size for the 2002 through 2009 high school cohorts.

\section{High School Graduation variables}

High school graduation variables were pulled from the 2002 through 2009 city and state graduation files. City files are available for all years, while state files are only available for $2002-2008$. In overlapping years the state files are given precendence. A student is defined as having graduated if she received a local, Regents, or Advanced Regents diploma. We define students who transfer, drop out, receive a GED, or receive a special education diploma as having not graduated.

\section{SAT variables}

SAT score variables were pulled from the 2006 through 2009 city SAT files, which consists of SAT scores for all students enrolled in a public NYC school when taking the SAT. We use a student's best combined score during the testing period. All scores are out of 2400 .

\subsection{Specialized High Schools Admissions Test Data}

Admissions to the academic SHS is determined by the Specialized High Schools admissions test (SHSAT). The test is broken into two sections, one math and one verbal, and students are given 2 hours and 30 minutes per section with no break. The verbal section is made up of 45 multiplechoice questions. 30 questions test reading comprehension, 10 questions test logical reasoning, and 5 questions require students to put sentences into the most logical order in a paragraph. The math section is comprised of 50 multiple-choice questions, which test basic math, algebra (factoring and substitution), geometry, basic graphing, logic, and word problems.

In scoring the SHSAT, there is no penalty for wrong answers and correct answers receive points. The raw number of questions correct is scaled through a Department of Education formula, and scores fall between 200 and 800. Due to the formula used by the DOE, students who score very 
highly in one section but poorly in another are more likely to have a higher score than students who score well on both.

On the day of the exam, students rank the schools in order of where they want to go. Test results are ranked from the highest score to the lowest, and administrators place students in high schools starting with the students with the highest score. Each student is placed into their most preferred school that still has seats until no seats remain at any SHS.

SHSAT scores, gender and middle school type were pulled from the NYC test score for 1989 through 2008. Scores were standardized by year to have mean of zero and standard deviation of one in the sample of test takers. Eligibility rank and score cutoffs were defined as the first student who wanted to attend a specific school but was not accepted to that school. Gender was coded as male, non-male, or missing. Middle school type was coded as public, private, or missing. We consider only eighth grade admissions, dropping all SHSAT scores from ninth grade.

\subsection{National Student Clearinghouse Data}

Information on college attendance and graduation comes from the National Student Clearinghouse (NSC), a non-profit organization that maintains enrollment information for 92 percent of colleges nationwide. We provided each student's full name, date of birth and high school graduation date, which the NSC used to match to its database. The NSC data contain information on enrollment spells for all covered colleges that a student attended. Information is available on full or part-time status and degree receipt in some cases.

We code a student as having enrolled in a four-year college if she ever attends a four-year school in the NSC data. We code a student as having graduated from a four-year college if the NSC data indicates him having received a degree from a four-year school. We code a student as having enrolled in a post-graduate university if they enroll in any non-two-year college after receiving a four-year degree.

To provide a measure of college quality, we match the NSC data to data on college characteristics from the U.S. News and World Report. The U.S. News and World Report collects data on college characteristics and statistics for four-year colleges in the U.S., including average class size, size of the faculty, graduation rates, tuition, room and board, average debt, loan size, percent of students receiving aid, acceptance rate, standardized test scores, high school GPA where available, demographic information on gender and the diversity index, freshman retention, and annual alumni donations. We use midpoint SAT score as our primary measure of college quality. When only ACT scores are available, we convert them to SAT scores using the ACT's official score concordance chart found at http://www.act.org/aap/concordance/. We code a student as having attended a school with an SAT over 1200/1300/1400 if any of the four-year schools attended by that student have a median SAT over that threshold. 